\title{
NEURONAL NETWORKS WITH GAP JUNCTIONS: A STUDY OF PIECE-WISE LINEAR PLANAR NEURON MODELS*
}

\author{
S COOMBES ${ }^{\dagger}$
}

\begin{abstract}
.
The presence of gap junction coupling among neurons of the central nervous systems has been appreciated for some time now. In recent years there has been an upsurge of interest from the mathematical community in understanding the contribution of these direct electrical connections between cells to large-scale brain rhythms. Here we analyze a class of exactly soluble single neuron models, capable of producing realistic action potential shapes, that can be used as the basis for understanding dynamics at the network level. This work focuses on planar piece-wise linear models that can mimic the firing response of several different cell types. Under constant current injection the periodic response and phase response curve (PRC) is calculated in closed form. A simple formula for the stability of a periodic orbit is found using Floquet theory. From the calculated PRC and the periodic orbit a phase interaction function is constructed that allows the investigation of phase-locked network states using the theory of weakly coupled oscillators. For large networks with global gap junction connectivity we develop a theory of strong coupling instabilities of the homogeneous, synchronous and splay state. For a piece-wise linear caricature of the Morris-Lecar model, with oscillations arising from a homoclinic bifurcation, we show that large amplitude oscillations in the mean membrane potential are organized around such unstable orbits.
\end{abstract}

Key words. piece-wise linear models, gap junctions, Floquet theory, coupled-oscillator theory, phase-density function

AMS subject classifications. 92C20

1. Introduction. Gap junctions allow for direct communication between cells. They are typically formed from the juxtaposition of two hemi-channels (connexin proteins) and allow the free movement of ions or molecules across the intercellular space separating the plasma membrane of one cell from another. Gap junction coupling is known to occur between many cell types, including for example pancreatic- $\beta$ cells [21], heart cells [23] and astrocytes [9]. It is no understatement to say that they are now believed to be ubiquitous throughout the central nervous system [16]. Indeed it has been appreciated for some time that they exist between inhibitory neurons of the neocortex [35]. As well as being found in neocortex $[36,4,39,34]$, they occur in many other brain regions, including the hippocampus [34], inferior olivary nucleus in the brain stem [75], the spinal cord [71], the thalamus [47] and have recently been shown to form axo-axonic connections between excitatory cells in the hippocampus (on mossy fibers) [41]. Without the need for receptors to recognize chemical messengers gap junctions are much faster than chemical synapses at relaying signals. The synaptic delay for a chemical synapse is typically in the range $1-100 \mathrm{~ms}$, while the synaptic delay for an electrical synapse may be only about $0.2 \mathrm{~ms}$. There is now little doubt that gap junctions play a substantial role in the generation of neural rhythms, both functional $[3,46,8]$ and pathological [82, 24], and that they may subserve system level computations [62].

The presence of gap-junctional coupling in a neuronal network necessarily means that neurons directly 'feel' the shape of action potentials (APs) from other neurons to which they are connected. From a modeling perspective one must therefore be careful to work with single neuron models that have an accurate representation of an AP shape. To date there is now a zoo of single neuron models that can accurately reflect these shapes for different neuronal cell types (see for example [49]). Typically such models, being based around that of Hodgkin-Huxley [44], are high dimensional and can often only be analyzed using perturbative techniques, such as geometric singular perturbation theory (see [72] for a review). When combined with an initial reduction of the model, say using the techniques in [54], this has proven a remarkably powerful approach for gaining insight into single neuron behavior. However, it does not necessarily pave the way for tractable network studies. In this case starting from a one dimensional integrate-and-fire (IF) type model is often advocated [11]. However, since the IF model does not generate an action potential shape it must be augmented in some way as in $[15,37]$, leading one naturally to consider the spike-response model [38]. However, in this case the AP is considered to have

*SIAM J. Appl. Dyn. Syst. (Submitted)

${ }^{\dagger}$ Department of Mathematical Sciences, University of Nottingham, Nottingham, NG7 2RD, UK 
a universal shape, triggered as the voltage reaches a constant voltage threshold. This does not quite capture the dynamics of a truly excitable system (with gating variables) where instead one would expect a state-dependent threshold and a variable AP shape. Thus we are naturally led to a search for planar models possessing one voltage and one gating variable that can mimic the behavior of high dimensional conductance based models. Perhaps the most famous example of such a model is the FitzHugh-Nagumo model [31], which has many of the same characteristics as the Hodgkin-Huxley model. In this case analytical progress has been possible with one further step, namely the introduction of piece-wise linear (PWL) nullclines. This gives rise to the so-called McKean model [64], for which a number of results about the existence and stability of periodic orbits are now known [83, 84]. In this paper we introduce a broader class of PWL models that can mimic the behavior of many common cell types and describe how to analyze periodic orbits explicitly. Importantly we show that the study of such models does indeed allow for mathematical studies of the rich dynamical behavior seen in large networks with strong gap junction coupling. In this sense our work is complementary to many other theoretical studies that focus on weak coupling $[74,69,7,61,56,26]$ as well as computational studies with strong coupling $[55,60,3,76]$.

One of the main motivations for pursuing the work in this paper is that it may underpin the development of a tractable firing rate model of neural tissue possessing gap junctions. Necessarily this must require an understanding of strong coupling if gap induced variations in firing rate are of interest. With the exception of work by van Vreeswijk [81] (for synaptic interactions) results for strong coupling are rare. Hence, although we focus on the special case of PWL neuron models this is useful as it allows for some specific insight to be gained into dynamics in the strong coupling regime. Moreover, some of the techniques we develop here, notably for determining the stability of the asynchronous state in a strongly gap-junction coupled global network, are valid not just for PWL systems but more general limit cycle oscillator networks. The more detailed structure of this paper is as follows. In section 2 we introduce the class of PWL models that we study throughout the paper. In particular we focus on two distinct examples, one of which is the McKean model and the other a new PWL model that caricatures the conductance-based Morris-Lecar model with oscillations generated via a homoclinic bifurcation [68]. Next, in section 3, we show how to analyze periodic orbits that arise in such models under constant current injection. This includes the construction of orbits, the determination of their stability, and the calculation of the phase response curve for the orbit. Stability is analyzed using Floquet theory and shown to lead to a simple formula for the non-zero Floquet exponent. Network studies are pursued in section 4 for two important cases: i) weak coupling, and ii) strong coupling. In the former case we show how to calculate the phase interaction function for a network in closed form using a Fourier representation. This is used to investigate phase-locked states in both small and large networks. Focusing on synchronous and splay states in globally coupled networks we further show how to treat the case of strong coupling. Our results for existence and stability recover those of the weak coupling theory in the appropriate limit. In section 5 we use this strong coupling theory to understand large amplitude oscillations seen in the mean field signal of networks of Morris-Lecar neurons with gap junction coupling [42]. Finally in section 6 we discuss natural extensions of the work in this paper.

2. Piece-wise linear neuron models. The excitable properties of neural cells can often be summarized simply by determining their firing rate response to constant current injection. Broadly speaking one then either classifies a neuron as being Type I or Type II. Type I is obtained when repetitive action potentials are generated with an arbitrarily low frequency, whereas in Type II action potentials emerge at a nonzero frequency. In this latter case it is natural to think of oscillations as arising through a Hopf bifurcation. Indeed from the seminal experimental and modeling work of Hodgkin and Huxley this is known to be the case for the squid giant axon. Thus, although the original Hodgkin-Huxley model consists of four nonlinear ordinary differential equations (ODEs) it is not surprising that alternative planar models can also be invoked to fit at least the firing rate response. The classic example being the FitzHugh-Nagumo model [31], though others such as obtained by a systematic reduction of the Hodgkin-Huxley equations are known [1]. These planar models are described by two coupled nonlinear ODEs, one for voltage and the other for a single effective gating variable. The nullcline for the voltage variable has a cubic shape typical of many excitable systems. Although powerful geometric techniques may be brought to bear on such planar models their analysis in closed form is precluded by the presence of the cubic nonlinearity. 


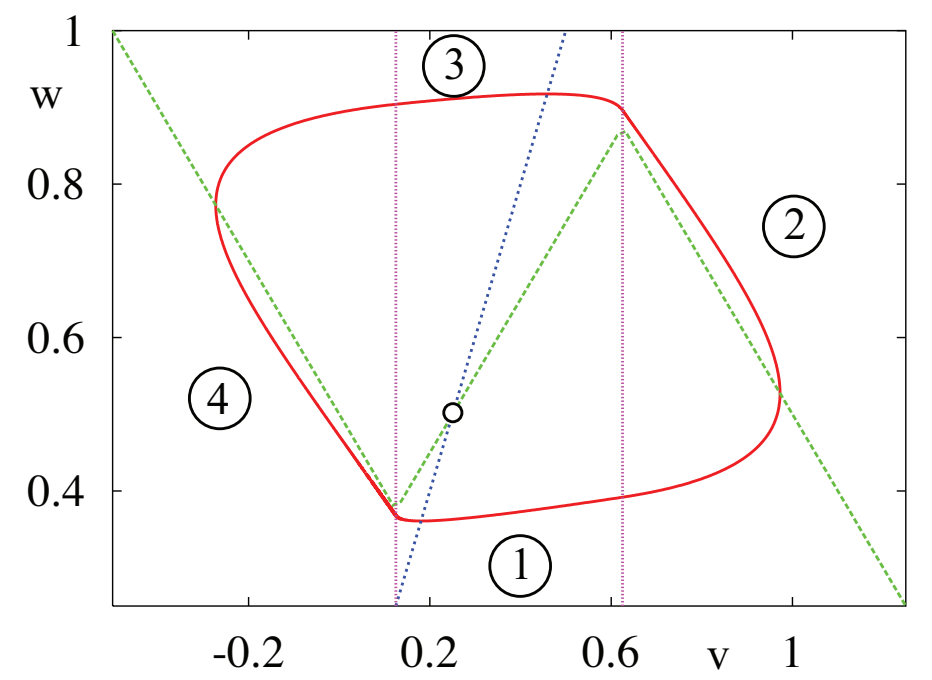

FIG. 2.1. The phase plane for the McKean model has a nullcline with a piece-wise linear cubic shape (dashed green line) corresponding to $\dot{v}=0$ and a linear one associated with $\dot{w}=0$ (dotted blue line). Parameters are $C=0.1, I=0.5, \gamma=0.5$, and $a=0.25$. The red line corresponds to a stable periodic orbit.

This has motivated the introduction and study of PWL caricatures, such as the McKean model [64, 79]. The equations for a single two-dimensional McKean neuron take the form

$$
\begin{aligned}
C \dot{v} & =f(v)-w+I, \\
\dot{w} & =g(v, w),
\end{aligned}
$$

where the functions $f(v)$ and $g(v, w)$ are given by

$$
\begin{aligned}
f(v) & = \begin{cases}-v, & v<a / 2 \\
v-a, & a / 2 \leq v \leq(1+a) / 2, \\
1-v, & v>(1+a) / 2\end{cases} \\
g(v, w) & =v-\gamma w .
\end{aligned}
$$

Here, $C>0, \gamma>0, I$ is a constant drive and $f(v)$ is a PWL caricature of the cubic FitzHugh-Nagumo nonlinearity $f(v)=v(1-v)(v-a)$, whilst $g(v, w)$ describes the linear dynamics of the gating variable. Another popular choice for $f(v)$ is the function $f(v)=-v+\Theta(v-a)$, where $\Theta$ is the Heaviside step function. The analysis of this latter nonlinearity has been pursued in detail by Tonnelier $[78,80]$. A phaseplane plot of the McKean model is shown in Fig. 2.1. To generate Type I behavior, often associated with either a homoclinic bifurcation or a saddle-node on an invariant cycle (SNIC) [27], necessarily requires the introduction of a nonlinear dynamics for the gating variable, as in the Morris-Lecar model or the cortical neuron model of Wilson [85]. A PWL idealization of the Morris-Lecar model has already been introduced by Tonnelier and Gerstner [80], and since the nullcline of the gating variable in the Wilson model has a quadratic shape, it too is easy to caricature. Indeed many of the shapes for $g(v, w)$ underlying a Type I response appear to be described with the simple continuous choice

$$
g(v, w)= \begin{cases}\left(v-\gamma_{1} w+b^{*} \gamma_{1}-b\right) / \gamma_{1}, & v<b \\ \left(v-\gamma_{2} w+b^{*} \gamma_{2}-b\right) / \gamma_{2}, & v \geq b\end{cases}
$$

with $-a / 2<b^{*}<(1-a) / 2$ and $a / 2<b<(1+a) / 2$. Here we take $\gamma_{2}>0$, though allow $\gamma_{1}$ to take both positive and negative values. Another natural choice, though this time discontinuous, is $g(v, w)=v-\gamma w+\Theta(v-b)$, which has been used to caricature the Morris-Lecar model in particular [80]. Note that (up to a constant shift) we recover the PWL McKean model with the choice $\gamma_{1}=\gamma=\gamma_{2}$ 


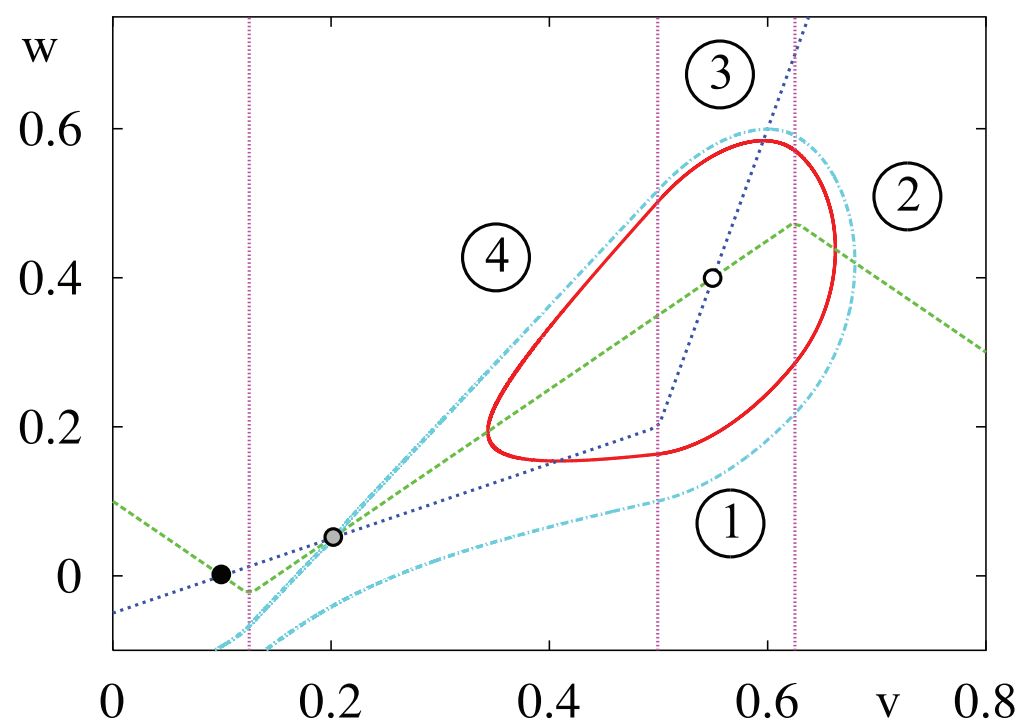

FIG. 2.2. The phase plane for the piece-wise linear Morris-Lecar (PML) model with $\gamma_{1}=2, \gamma_{2}=0.25, C=0.825$, $I=0.1, a=0.25$ and $b=0.5$ and $b^{*}=0.2$. The pale blue line passing through the saddle (gray filled circle) is the separatrix between the stable fixed point (black filled circle) and the stable limit cycle (in red).

in equation (2.5). An example with dynamics that is bistable between a fixed point and a limit cycle is shown in Fig. 2.2. Here the emergence of low frequency oscillations is associated with a homoclinic bifurcation, whereby the amplitude of the periodic orbit grows with a decrease in $I$ and collides with a saddle point. We regard this model as a PWL caricature of the Morris-Lecar neuron, with oscillations arising from a homoclinic bifurcation, and as such shall refer to it as the PML model. On a technical point it is important to note that it is not possible to have a smooth SNIC with a piece-wise linear model, since it would not contain any quadratic parts (necessary to define a saddle-node bifurcation). One such example, would be the nonlinear integrate-and-fire neuron, described by Karbowski and Kopell [52] with subthreshold dynamics $\dot{v}=|v|+I$. Throughout the rest of this paper we shall work with the PWL model defined by equations (2.3) and (2.5), though we stress here that the techniques we develop work for all of the PWL choices for $f(v)$ and $g(v, w)$ that we have discussed above.

3. Periodic orbits. As a PWL planar system much can be said about the dynamics of models defined by equations (2.3) and (2.5). For the special case that $f(v)=-v+\Theta(v-a)$ and $g(v, w)=v$ Tonnelier [78] has shown how to use the method of harmonic balance [2] to obtain information about periodic orbits. Here we present an alternative approach that can tackle more general choices for $f$ and $g$. In essence we solve the system in each of its linear regimes and demand continuity of solutions to construct orbits of the full nonlinear flow. To see how we do this it is first convenient to consider a two-dimensional linear system of the form

$$
\dot{z}=A z+b, \quad z=\left[\begin{array}{c}
v \\
w
\end{array}\right]
$$

where the $2 \times 2$ matrix $A$ has components $a_{i j}, i, j=1,2$, and $b$ is a constant $2 \times 1$ input vector. The solution to (3.1) may be written in the form

$$
z(t)=G(t) z(0)+K(t) b, \quad G(t)=\mathrm{e}^{A t}, \quad K(t)=\int_{0}^{t} G(s) \mathrm{d} s .
$$

If $A$ has real eigenvalues $\lambda_{ \pm}$, such that $A q_{ \pm}=\lambda_{ \pm} q_{ \pm}$with $q_{ \pm} \in \mathbb{R}^{2}$, given by

$$
\lambda_{ \pm}=\frac{\operatorname{Tr} A \pm \sqrt{(\operatorname{Tr} A)^{2}-4 \operatorname{det} A}}{2},
$$


then we may 'diagonalize' and write $G(t)$ in the computationally useful form $G(t)=P \mathrm{e}^{\Lambda t} P^{-1}$, where $\Lambda=\operatorname{diag}\left(\lambda_{+}, \lambda_{-}\right), P=\left[q_{+}, q_{-}\right]$, and $q_{ \pm}=\left[\left(\lambda_{ \pm}-a_{22}\right) / a_{21}, 1\right]^{T}$. If $A$ has complex eigenvalues $\rho \pm i \omega$ then the associated complex eigenvector is $q$ such that $A q=(\rho+i \omega) q, q \in \mathbb{C}^{2}$. In this case $G(t)=\mathrm{e}^{\rho t} P \mathcal{R}_{\omega t} P^{-1}$, where

$$
\mathcal{R}_{\theta}=\left[\begin{array}{cc}
\cos \theta & -\sin \theta \\
\sin \theta & \cos \theta
\end{array}\right], \quad P=[\operatorname{Im}(q), \operatorname{Re}(q)]=\left[\begin{array}{cc}
0 & 1 \\
\widehat{\omega} & \widehat{\rho}
\end{array}\right],
$$

with $\widehat{\omega}=\omega / a_{12}$ and $\widehat{\rho}=\left(\rho-a_{11}\right) / a_{12}$. Note that $\rho$ and $\omega$ may be written using the invariance of Tr and det as $\rho=\left(a_{11}+a_{22}\right) / 2, \omega^{2}=a_{11} a_{22}-a_{12} a_{21}-\rho^{2}>0$. The explicit form for $G(t)$, necessary for carrying out computations, is given in Appendix A.

To specify a periodic orbit of the PWL model of choice it is convenient to break the solution into pieces such that on each piece the dynamics is governed by a linear dynamical system. As a concrete example we will focus on the type of periodic orbits shown in Figs. 2.1 and 2.2. In both these examples we need only consider four distinct pieces, labeled by $\mu=1, \ldots, 4$. We denote the time spent in each of these four states as $T_{\mu}$. For each piece we write $z_{\mu}(t)=G_{\mu}(t) z_{\mu}(0)+K_{\mu}(t) b_{\mu}$ with the forms for $G_{\mu}$ and $K_{\mu}$ given by (3.2) under the replacement of $A$ by $A_{\mu}$. For the McKean model we have that $A_{1}=A_{3}$, $A_{2}=A_{4}$ where

$$
A_{1}=\left[\begin{array}{cc}
1 / C & -1 / C \\
1 & -\gamma
\end{array}\right], \quad A_{2}=\left[\begin{array}{cc}
-1 / C & -1 / C \\
1 & -\gamma
\end{array}\right]
$$

with

$$
b_{1}=\left[\begin{array}{c}
(I-a) / C \\
0
\end{array}\right], \quad b_{2}=\left[\begin{array}{c}
(1+I) / C \\
0
\end{array}\right], \quad b_{4}=\left[\begin{array}{c}
I / C \\
0
\end{array}\right],
$$

and $b_{3}=b_{1}$. For the PML model defined by $(2.5)$

$$
A_{1}=\left[\begin{array}{cc}
1 / C & -1 / C \\
1 / \gamma_{2} & -1
\end{array}\right], \quad A_{2}=\left[\begin{array}{cc}
-1 / C & -1 / C \\
1 / \gamma_{2} & -1
\end{array}\right], \quad A_{4}=\left[\begin{array}{cc}
1 / C & -1 / C \\
1 / \gamma_{1} & -1
\end{array}\right]
$$

with

$$
b_{1}=\left[\begin{array}{c}
(I-a) / C \\
b^{*}-b / \gamma_{2}
\end{array}\right], \quad b_{2}=\left[\begin{array}{c}
(1+I) / C \\
b^{*}-b / \gamma_{2}
\end{array}\right], \quad b_{4}=\left[\begin{array}{c}
(I-a) / C \\
b^{*}-b / \gamma_{1}
\end{array}\right],
$$

and $A_{3}=A_{1}$ and $b_{3}=b_{1}$. Introducing two voltage thresholds $v_{\text {th }}^{1}$ and $v_{\text {th }}^{2}$, where $\left(v_{\mathrm{th}}^{1}, v_{\mathrm{th}}^{2}\right)=(a / 2,(1+$ a) $/ 2$ ) for the McKean model and $\left(v_{\mathrm{th}}^{1}, v_{\mathrm{th}}^{2}\right)=(b,(1+a) / 2)$ for the PML model, means that we can parameterize a periodic orbit by choosing initial data such that $z_{1}(0)=\left(v_{\mathrm{th}}^{1}, w^{*}\right)$ (with $w^{*}$ as yet undetermined) and $z_{\mu+1}(0)=G_{\mu}\left(T_{\mu}\right) z_{\mu}(0)+K_{\mu}\left(T_{\mu}\right) b_{\mu}$, for $\mu=1,2,3$. The 'times-of-flight' $T_{\mu}$ are determined by solving the threshold crossing conditions: $v_{1}\left(T_{1}\right)=v_{\mathrm{th}}^{2}, v_{2}\left(T_{2}\right)=v_{\mathrm{th}}^{2}, v_{3}\left(T_{3}\right)=v_{\mathrm{th}}^{1}$, and $v_{4}\left(T_{4}\right)=v_{\mathrm{th}}^{1}$. A periodic solution can then be found by solving $w_{4}\left(T_{4}\right)=w_{1}(0)$, thus yielding $w^{*}$ and the period $T=\sum_{\mu=1}^{4} T_{\mu}$. In Fig. 3.1 we plot the period and orbit shape as a function of the external drive $I$ obtained using the prescription above. A similar plot for the PML model is shown in Fig. 3.2. In contrast to the McKean model the firing rate of the PML model at the onset of repetitive behavior increases from zero, as expected for a system with a homoclinic bifurcation.

Three other types of periodic solution are also possible. Two of these involve only a single threshold crossing. Namely one which crosses through the section $v=v_{\text {th }}^{1}$, but not $v=v_{\text {th }}^{2}$ and another which crosses through $v=v_{\mathrm{th}}^{2}$, but not $v=v_{\mathrm{th}}^{1}$. Calling these the $s u b$ - and supra-threshold periodic orbits respectively then each may also be solved for using the approach (and notation) above. The sub-threshold orbit is specified by the restriction $\mu=\{1,4\}$ with $z_{1}(0)=\left(v_{\mathrm{th}}^{1}, w^{*}\right)$, subject to $v_{1}\left(T_{1}\right)=v_{\text {th }}^{1}=v_{4}\left(T_{4}\right)$ and $w_{4}\left(T_{4}\right)=w_{1}(0)$, so that $T=T_{1}+T_{4}$. The supra-threshold orbit is specified by the restriction $\mu=\{2,3\}$ with $z_{2}(0)=\left(v_{\mathrm{th}}^{2}, w^{*}\right)$, subject to $v_{2}\left(T_{2}\right)=v_{\mathrm{th}}^{2}=v_{3}\left(T_{3}\right)$ and $w_{3}\left(T_{3}\right)=w_{2}(0)$, so that $T=T_{2}+T_{3}$. Examples of such orbits are shown in Fig. 3.3. The final type of orbit does not cross any thresholds, and is defined by simply by $v(T)=v_{\text {th }}$ and $v(T)=v(0)$ for some section $v_{\text {th }}$ through the orbit. We shall call such an orbit harmonic, because its shape will be determined by a linear system of ODEs. Note however that it will only exist at an isolated point in parameter space, namely where the coefficient matrix $A$ has purely complex eigenvalues ( $\operatorname{Tr} A=0, \operatorname{det} A>0)$. 

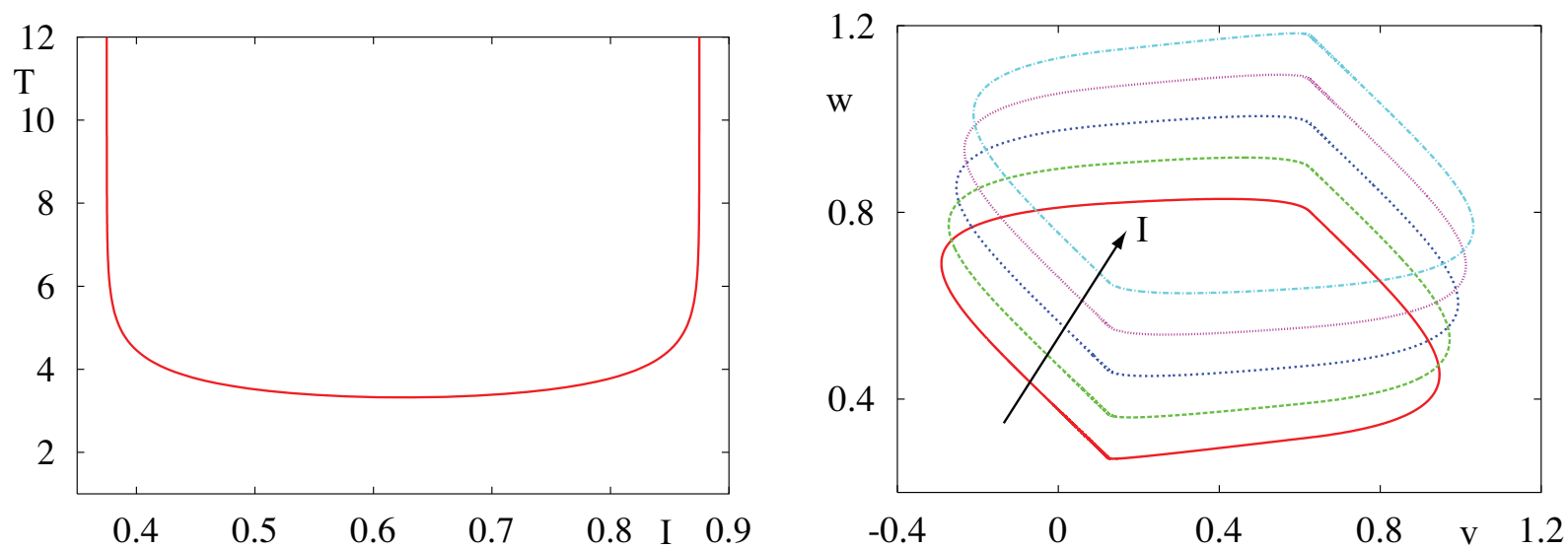

FIG. 3.1. McKean model. Left: period of solution as a function of background drive I. Right: shape of orbits for $I=0.4,0.5,0.6,0.7,0.8$. Other parameters as in Fig. 2.1.
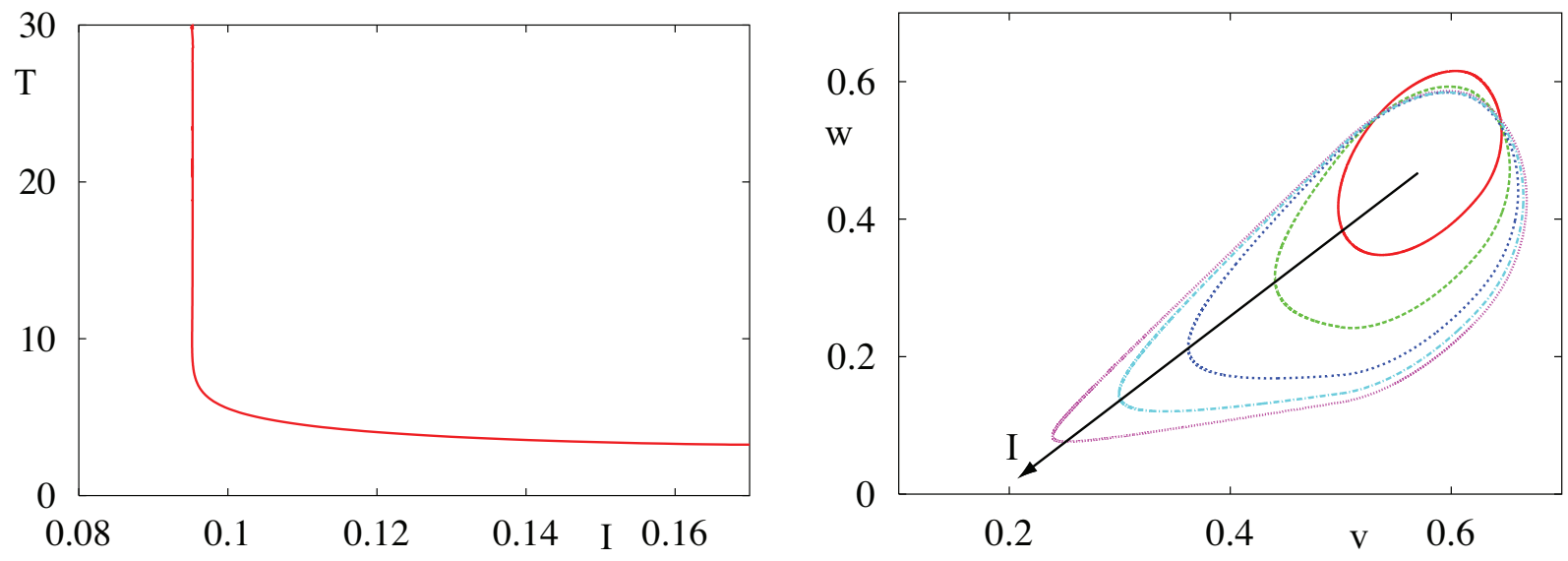

FIG. 3.2. PML model. Left: period of solution as a function of background drive I. Right: shape of orbits for I ranging from 0.17 to 0.09 . Other parameters as in Fig. 2.2.
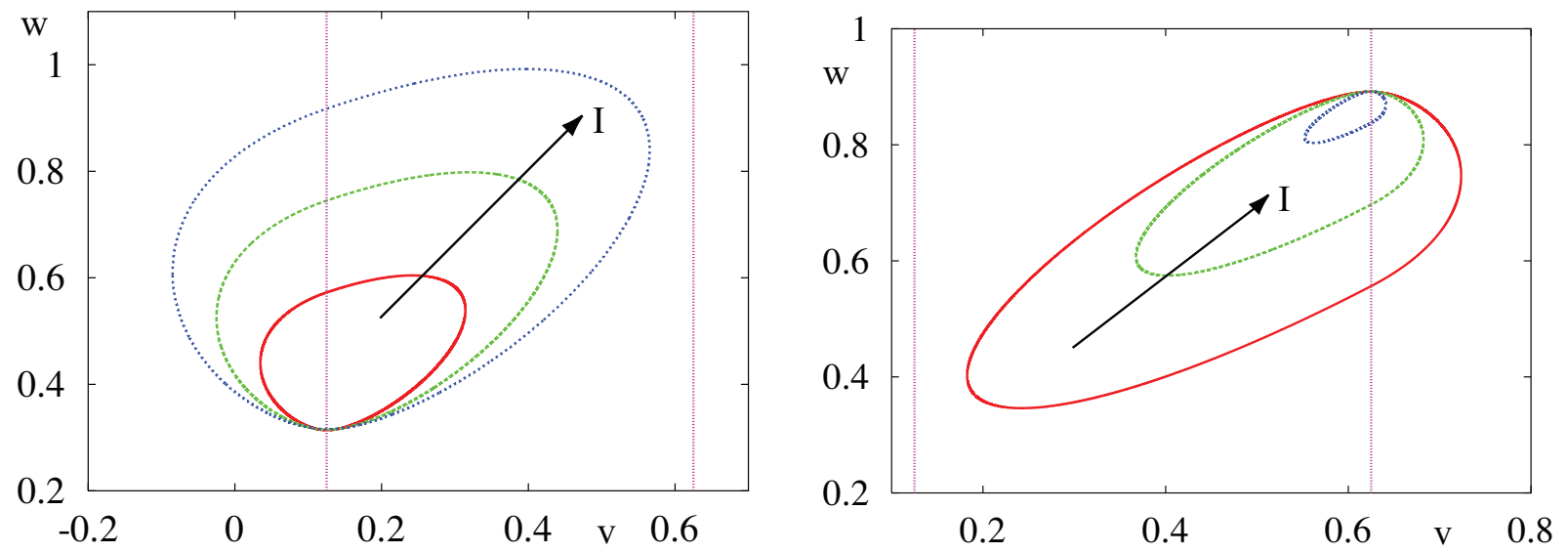

FIG. 3.3. McKean model. Left: Sub-threshold orbits with $C=1, \gamma=0.4$, and $I=0.475,0.5,0.525$. All these subthreshold orbits have a common period. Right: Supra-threshold orbits with $C=1, \gamma=0.7$, and $I=0.47,0.49,0.51$. All these super-threshold orbits have a common period. Other parameters as in Fig. 2.1. 
3.1. Phase response curve. It is common practice in neuroscience to characterize a neuronal oscillator in terms of its phase response to a perturbation. This gives rise to the notion of a so-called phase response curve (PRC). For a detailed discussion of PRCs we refer the reader to [29, 30, 45]. Suffice to say that there are three main ways to calculate PRCs, attributed to Winfree, Kuramoto and Malkin. A nice comparison of these three approaches can be found in [50]. For concreteness we shall follow the exposition in [13] for the Malkin adjoint method. Consider a dynamical system $\dot{z}=F(z)$ with a $T$ periodic solution $Z(t)=Z(t+T)$ and introduce an infinitesimal perturbation $\Delta z_{0}$ to the trajectory $Z(t)$ at time $t=0$. This perturbation evolves according to the linearized equation of motion:

$$
\frac{\mathrm{d} \Delta z}{\mathrm{~d} t}=D F(Z(t)) \Delta z, \quad \Delta z(0)=\Delta z_{0} .
$$

Here $D F(Z)$ denotes the Jacobian of $F$ evaluated along $Z$. Introducing a time-independent phase shift $\Delta \theta$ as $\theta(Z(t)+\Delta z(t))-\theta(Z(t))$ we have to first order in $\Delta z$ that

$$
\Delta \theta=\langle Q(t), \Delta z(t)\rangle,
$$

where $\langle\cdot, \cdot\rangle$ defines the standard inner product, and $Q=\nabla_{Z} \theta$ is the gradient of $\theta$ evaluated at $Z(t)$. Taking the time-derivative of (3.10), gives

$$
\left\langle\frac{\mathrm{d} Q}{\mathrm{~d} t}, \Delta z\right\rangle=-\left\langle Q, \frac{\mathrm{d} \Delta z}{\mathrm{~d} t}\right\rangle=-\langle Q, D F(Z) \Delta z\rangle=-\left\langle D F^{T}(Z) Q, \Delta z\right\rangle .
$$

Since the above equation must hold for arbitrary perturbations we see that the gradient $Q=\nabla_{Z} \theta$ satisfies the linear equation

$$
\frac{\mathrm{d} Q}{\mathrm{~d} t}=D(t) Q, \quad D(t)=-D F^{T}(Z(t)),
$$

subject to the conditions $\nabla_{Z(0)} \theta \cdot F(Z(0))=1 / T$ and $Q(t)=Q(t+T)$. The first condition simply guarantees that $\dot{\theta}=1 / T$ (at any point on the periodic orbit), and the second enforces periodicity. The (vector) PRC, $R$, is related to $Q$ according to the simple scaling $R=Q T$. In general (3.12) must be solved numerically to obtain the PRC, say using the adjoint routine in XPP [25]. However, for PWL models $D F(Z)$ is piece-wise constant and we can obtain a solution in closed form. Introducing a labeling as for the periodic orbit in section 3 we re-write (3.12) in the form $\dot{Q}_{\mu}=D_{\mu} Q_{\mu}$, where $D_{\mu}=-A_{\mu}^{T}$. The solution of each subsystem is given by $Q_{\mu}(t)=G_{\mu}^{T}\left(T_{\mu}-t\right) Q_{\mu}\left(T_{\mu}\right)$ with $Q_{\mu}\left(T_{\mu}\right)=Q_{\mu+1}(0)$, for $\mu=1,2,3$. Denoting $Q_{4}\left(T_{4}\right)=\left(q_{1}, q_{2}\right)$ we have the relation

$$
\frac{q_{1}}{\mu}\left[f\left(v_{\mathrm{th}}^{1}\right)-w^{*}+I\right]+q_{2} g\left(v_{\mathrm{th}}^{1}, w^{*}\right)=\frac{1}{T} .
$$

Periodicity is ensured by choosing $Q_{1}(0)=Q_{4}\left(T_{4}\right)$. After introducing the $2 \times 2$ matrix $\Gamma=G_{1}^{T}\left(T_{1}\right) G_{2}^{T}\left(T_{2}\right) G_{3}^{T}\left(T_{3}\right) G_{4}^{T}\left(T_{4}\right)$, this periodicity condition takes the form

$$
\left(\Gamma_{11}-1\right) q_{1}+\Gamma_{12} q_{2}=0 .
$$

Hence (3.13) and (3.14) define a pair of linear equations for $\left(q_{1}, q_{2}\right)$ that we may write in the form

$$
\Psi\left[\begin{array}{l}
q_{1} \\
q_{2}
\end{array}\right]=\left[\begin{array}{c}
1 / T \\
0
\end{array}\right], \quad \Psi=\left[\begin{array}{cc}
\left(f\left(v_{\mathrm{th}}^{1}\right)-w^{*}+I\right) / \mu & g\left(v_{\mathrm{th}}^{1}, w^{*}\right) \\
\Gamma_{11}-1 & \Gamma_{12}
\end{array}\right] .
$$

This is easily solved, with say Cramer's rule, giving $q_{i}=\operatorname{det}\left(\Psi_{i}\right) / \operatorname{det}(\Psi)$, where

$$
\Psi_{1}=\left[\begin{array}{cc}
1 / T & g\left(v_{\mathrm{th}}^{1}, w^{*}\right) \\
0 & \Gamma_{12}
\end{array}\right], \quad \Psi_{2}=\left[\begin{array}{cc}
\left(f\left(v_{\mathrm{th}}^{1}\right)-w^{*}+I\right) / \mu & 1 / T \\
\Gamma_{11}-1 & 0
\end{array}\right] .
$$

Similarly we may also construct the PRCs for the sub- and supra-threshold orbits (though we omit the details here). Note that the discussion above assumes that the underlying dynamical system is described 

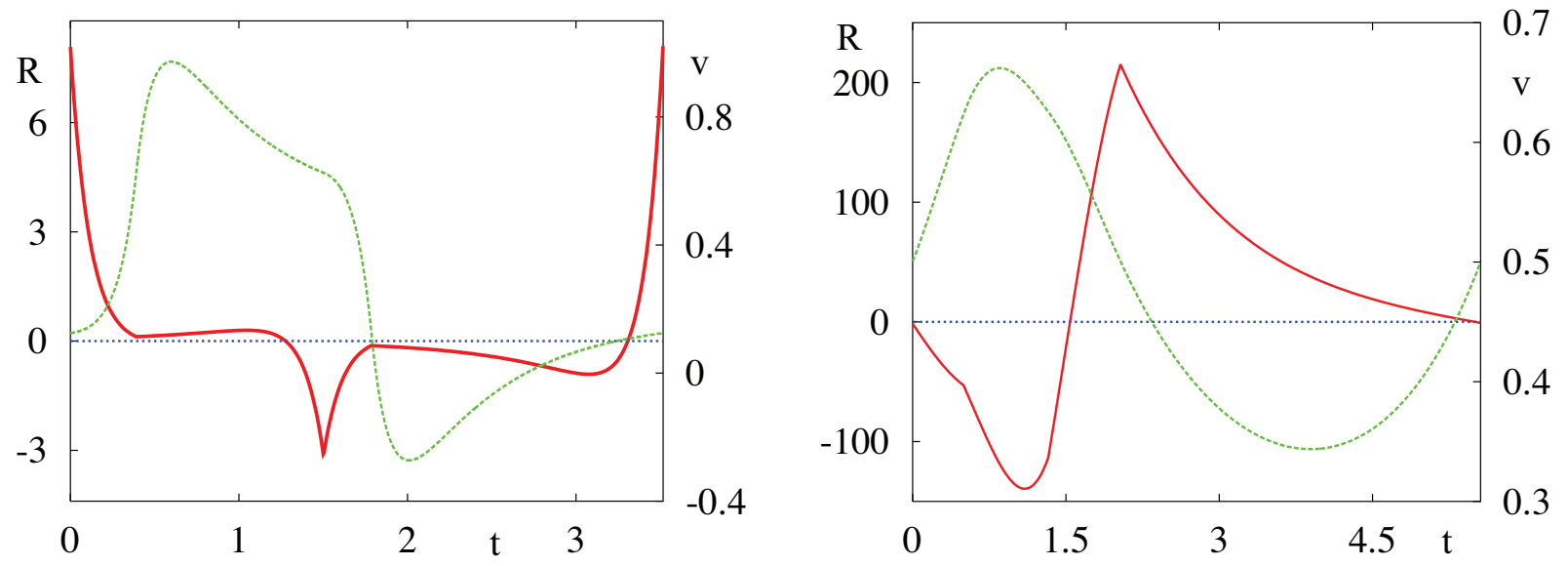

FIG. 3.4. Phase response curve (first component of $Q(t)$ scaled by $T$ ). The dashed line shows the underlying shape of the periodic voltage solution. Left: McKean model PRC with parameters as in Fig. 2.1. Right: PML model with parameters as in Fig. 2.2.

by a continuous vector field, so that we are free to choose any point on the orbit to fix the condition $\dot{\theta}=1 / T$. For discontinuous systems such as would arise in the singular limit $C=0$ or with a discontinuous choice of $g(v, w)$ then conditions (3.13) and (3.14) are not sufficient. Techniques for tackling relaxation style oscillations that arise in the former case have been developed in [48, 17], whilst the latter case can easily be treated by writing down the matching conditions to fix $\dot{\theta}=1 / T$ at any jump discontinuities in $g(v, w)$. A plot of two example PRCs constructed using the above approach are shown in Fig. 3.4.

3.2. Stability: Floquet theory. The natural way to determine the stability of a periodic orbit is to use Floquet theory (see for example [14]). The linearized equations describing the evolution of perturbations around the periodic orbit are given by (3.9). Note that with the use of a time-ordering operator $\mathcal{T}$ we may write the fundamental matrix solution of this $T$-periodic system as

$$
G(t)=\mathcal{T}\left\{\exp \left[\int_{0}^{t} D F(Z(s)) \mathrm{d} s\right]\right\}
$$

where $\mathcal{T} D(t) D(s)=\Theta(t-s) D(t) D(s)+\Theta(s-t) D(s) D(t)$. Let $\mu_{k}$ be the (distinct) eigenvalues of $G(T)$ and write $\sigma_{k}=\ln \left(\mu_{k}\right) / T \bmod 2 \pi i$. It follows that the periodic orbit will be stable if all the Floquet exponents have negative real part, namely $\operatorname{Re} \sigma_{k}<0$ for all $k=1,2$. Note that one of the Floquet exponents is always zero since it corresponds to perturbations along the periodic orbit (i.e. $\dot{Z}$ is a solution of (3.9) with a Floquet multiplier equal to unity). For PWL models time-ordering is not an issue (since $D F$ is piece-wise constant) and we have that $G(T)=G_{4}\left(T_{4}\right) G_{3}\left(T_{3}\right) G_{2}\left(T_{2}\right) G_{1}\left(T_{1}\right)=\Gamma^{T}$. where

We now make use of the well known result $\mu_{1} \mu_{2}=\exp \left(\int_{0}^{T} \operatorname{Tr} D F(s) \mathrm{d} s\right)$ to obtain $\left(\sigma_{1}, \sigma_{2}\right)=(0, \sigma)$,

$$
\sigma=\frac{1}{T} \sum_{\mu=1} T_{\mu} \operatorname{Tr} A_{\mu}
$$

The non-zero exponent for the sub- and supra-threshold orbit is given by (3.18) with $T_{2}=T_{3}=0$ and $T_{1}=T_{4}=0$ respectively. For a harmonic splay state we have simply that $\sigma=\operatorname{Tr} A_{1}$. Periodic solutions are stable if $\sigma<0$. For example, a periodic solution of the McKean model has a non-zero Floquet exponent $\sigma=\left(T_{1}-T_{2}+T_{3}-T_{4}\right) /(C T)-\gamma$. Note that in the singular limit $C \rightarrow 0$ we expect $T_{1,3} \rightarrow 0$, so that $\sigma \leq 0$. Hence, any periodic orbits that persist in this limit will be stable. For the PML model $\sigma=\left(T_{1}-T_{2}+T_{3}+T_{4}\right) /(C T)-1$. Some example plots of the non-zero Floquet exponent as a function of the external drive $I$ are shown in Fig. 3.5. 

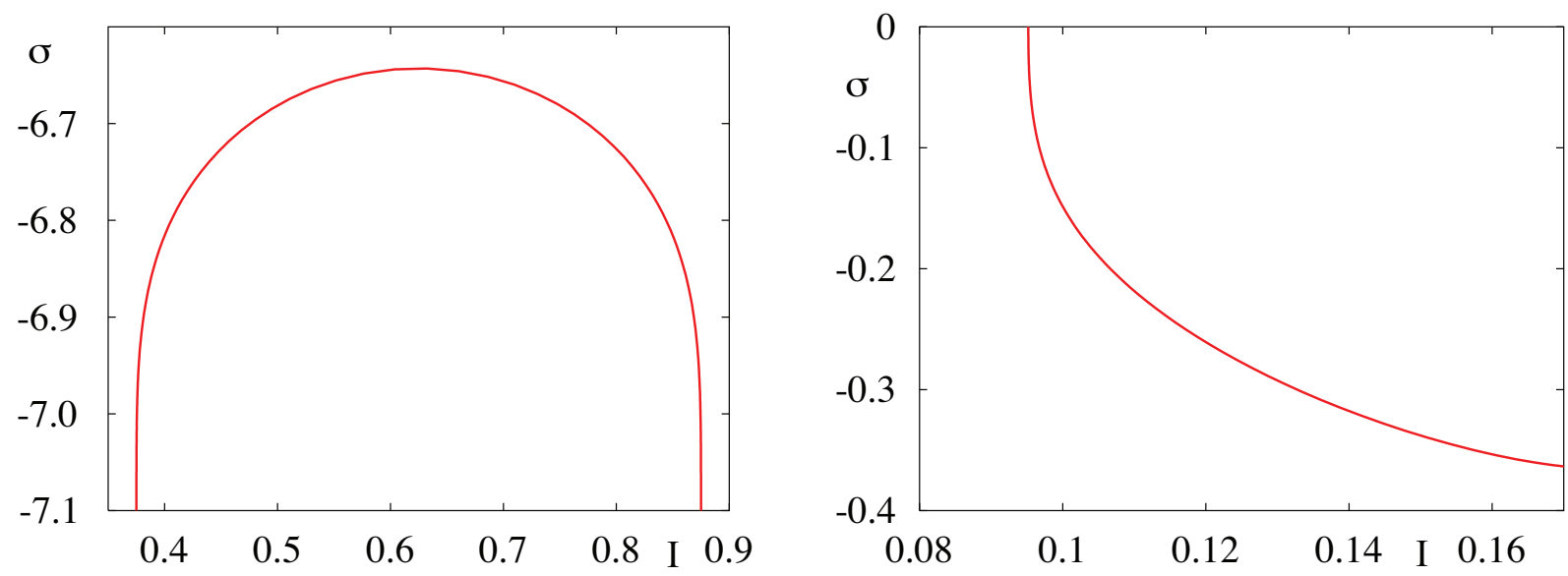

FIG. 3.5. Plot of the Floquet exponent $\sigma$. Left: Result for the McKean model using the solution branch of Fig. 3.1 (left). Right: Result for the PML model using the solution branch of Fig. 3.2 (left). Since $\sigma<0$ the solution branches in these two examples are stable.

4. Gap junction coupling. To model the direct gap junction coupling between two cells, one labeled post and the other pre, we introduce an extra current to the right hand side of (2.1) of the form

$$
I_{\text {gap }}=g_{\text {gap }}\left(v_{\text {pre }}-v_{\text {post }}\right)
$$

where $g_{\text {gap }}$ is the conductance of the gap junction. Indexing neurons in a network with the label $i=$ $1, \ldots, N$ and defining a gap junction conductance strength between neurons $i$ and $j$ as $g_{i j}$ means that neuron $i$ experiences a drive of the form $N^{-1} \sum_{j=1}^{N} g_{i j}\left(v_{j}-v_{i}\right)$. For a phase locked state then $z_{i}(t)=z(t-$ $\left.\phi_{i} T\right), z(t)=z(t+T)$, (for some constant phases $\left.\phi_{i} \in[0,1)\right)$ and we have $N$ equations distinguished by the driving terms $N^{-1} \sum_{j=1}^{N} g_{i j}\left(v\left(t+\left(\phi_{i}-\phi_{j}\right) T\right)-v(t)\right)$. In this section we pursue two approaches for studying networks of identical PWL neurons with such coupling terms. The first is the more familiar coupled oscillator approach, valid for weak coupling. The second approach exploits a Fourier representation to obtain closed form solutions for splay states with arbitrary coupling strength.

4.1. Weak coupling. The theory of weakly coupled oscillators $[57,28]$ is now a standard tool of dynamical systems theory and has been invoked by several authors to study networks with gap junctions $[69,61,70,26,63,53]$. It has also previously been used to study networks of McKean neurons in the singular limit $C \rightarrow 0[17,22]$. We introduce a time-dependent phase along the $T$-periodic orbit of an uncoupled neuron such that $\dot{\theta}_{i}(t)=1 / T$ for $i=1, \ldots, N$, with $\theta_{i} \in[0,1)$. In the presence of weak coupling (small $g_{i j}$ ), the dynamics for a gap junction coupled network then takes the form

$$
\frac{\mathrm{d} \theta_{i}}{\mathrm{~d} t}=\frac{1}{T}+\frac{1}{N} \sum_{j=1}^{N} g_{i j} H\left(\theta_{j}-\theta_{i}\right), \quad i=1, \ldots, N,
$$

where $H(\theta)$ is the so-called phase interaction function. For gap junction coupling this is given by

$$
H(\theta)=\frac{1}{T} \int_{0}^{T} Q^{T}(t)(v(t+\theta T)-v(t), 0) \mathrm{d} t,
$$

where $v(t)$ is a periodic solution of $(2.1)$ and $(2.2)$, and $Q(t)$ is the associated adjoint. It is convenient to introduce Fourier series for the $2 \times 1$ vectors $z$ and $Q$ and write

$$
z(t)=\sum_{n} z_{n} \mathrm{e}^{2 \pi i n t / T}, \quad Q(t)=\sum_{n} Q_{n} \mathrm{e}^{2 \pi i n t / T} .
$$


The phase interaction function then has the series representation:

$$
H(\theta)=\sum_{n} R_{n} v_{-n}\left[\mathrm{e}^{-2 \pi i n \theta}-1\right]
$$

where $v_{n}$ denotes the first component of $z_{n}$ and $R_{n}$ is the first component of $Q_{n}$. The Fourier coefficients $z_{n}$ and $Q_{n}$ may be obtained in closed form by taking Fourier transforms of the solutions for $z(t)$ and $Q(t)$. A straightforward calculation, using the forms of $z(t)$ and $Q(t)$ derived in sections 3 and 3.1, gives

$$
z_{n}=\frac{1}{T} \sum_{\mu=1}^{4}\left[\alpha_{\mu}^{n} z_{\mu}(0)+\gamma_{\mu}^{n} b_{\mu}\right] \mathrm{e}^{-2 \pi i n \nu_{\mu}}, \quad Q_{n}=\frac{1}{T} \sum_{\mu=1}^{4} \beta_{\mu}^{n} Q_{\mu}\left(T_{\mu}\right) \mathrm{e}^{-2 \pi i n \nu_{\mu}},
$$

where $\left(\nu_{1}, \nu_{2}, \nu_{3}, \nu_{4}\right)=\left(0, T_{1}, T_{1}+T_{2}, T_{1}+T_{2}+T_{3}\right) / T$ and the coefficients $\alpha_{\mu}^{n}, \beta_{\mu}^{n}$ and $\gamma_{\mu}^{n}$ are given explicitly by

$$
\alpha_{\mu}^{n}=\int_{0}^{T_{\mu}} G_{\mu}(t) \mathrm{e}^{-2 \pi i n t / T} \mathrm{~d} t, \quad \beta_{\mu}^{n}=\int_{0}^{T_{\mu}} G_{\mu}^{T}\left(T_{\mu}-t\right) \mathrm{e}^{-2 \pi i n t / T} \mathrm{~d} t, \quad \gamma_{\mu}^{n}=\int_{0}^{T_{\mu}} K_{\mu}(t) \mathrm{e}^{-2 \pi i n t / T} \mathrm{~d} t .
$$

Computationally useful forms for these matrix coefficients are given in Appendix B. Writing $H(\theta)$ as the Fourier series $\sum_{n} H_{n} \mathrm{e}^{2 \pi i n \theta}$ we have that $H_{n}=R_{-n} v_{n}$ for $n \neq 0$ and $H_{0}=-\sum_{n \neq 0} H_{n}$. From the structure of (4.7) given in Appendix B we see that the Fourier coefficients for the orbit and the response function decay as $1 / n$, and hence those of the phase interaction function as $1 / n^{2}$. Examples of phase interaction functions constructed using the above prescription are shown in Fig. 4.1.

We define a phase-locked solution to be of the form $\theta_{i}(t)=\phi_{i}+\Omega t$, where $\phi_{i}$ is a constant phase and $\Omega$ is the collective frequency of the coupled oscillators. Substitution into the averaged system (4.2) gives

$$
\Omega=\frac{1}{T}+\frac{1}{N} \sum_{j=1}^{N} g_{i j} H\left(\phi_{j}-\phi_{i}\right), \quad i=1, \ldots, N .
$$

After choosing some reference oscillator, these $N$ equations determine the collective frequency $\Omega$ and $N-1$ relative phases. In order to analyze the local stability of a phase-locked solution $\Phi=\left(\phi_{1}, \ldots, \phi_{N}\right)$, we linearize the system by setting $\theta_{i}(t)=\phi_{i}+\Omega t+\widetilde{\theta}_{i}(t)$ and expand to first-order in $\widetilde{\theta}_{i}$ to obtain:

$$
\frac{\mathrm{d} \widetilde{\theta}_{i}}{\mathrm{~d} t}=\frac{1}{N} \sum_{j=1}^{N} \widehat{\mathcal{H}}_{i j}(\Phi) \widetilde{\theta}_{j}, \quad \widehat{\mathcal{H}}_{i j}(\Phi)=g_{i j} H^{\prime}\left(\phi_{j}-\phi_{i}\right)-\delta_{i, j} \sum_{k=1}^{N} g_{i k} H^{\prime}\left(\phi_{k}-\phi_{i}\right),
$$

where $H^{\prime}(\phi)=\mathrm{d} H(\phi) / \mathrm{d} \phi$. One of the eigenvalues of the Jacobian $\widehat{\mathcal{H}}$ is always zero, and the corresponding eigenvector points in the direction of the flow, that is $(1,1, \ldots, 1)$. The phase-locked solution will be stable provided that all other eigenvalues have a negative real part. For two neurons, with $g_{i j}=g$, a phase locked state is defined by $G(\phi)=0$ where $G(\phi)=g[H(-\phi)-H(\phi)]$ and $\phi$ is the relative phase between the two. The condition for stability is simply $G^{\prime}(\phi)<0$. By symmetry the phase locked state $(\phi=0)$ and the anti-synchronous state $(\phi=1 / 2)$ are guaranteed to exist. In Fig. 4.2 we plot $G(\phi)$ for the phase interaction functions of Fig. 4.1. In this example we see that the McKean model admits a stable synchronous solution, whilst the PML model admits a stable anti-synchronous solution.

For globally coupled networks with $g_{i j}=g$ the system (4.2) is $\mathbf{S}_{N} \times \mathbf{T}^{1}$ equivariant. By the equivariant branching lemma maximally symmetric solutions describing synchronous, splay, and cluster states are expected to be generic [5]. For the synchronous state, defined by $\phi_{i}(t)=0$, the collective frequency is given simply as $\Omega=1 / T$, and $\widehat{\mathcal{H}}_{i j}(\Phi)=g H^{\prime}(0)\left[1-N \delta_{i j}\right]$. Hence, there is a single zero eigenvalue and an eigenvalue $\lambda=-g H^{\prime}(0)$ of multiplicity $N-1$. For the examples in Fig. 4.1 we see that the McKean model has a stable synchronous solution whilst the PML model, with oscillations generated by a homoclinic bifurcation, does not. If the underlying single neuron model has an oscillation generated by a SNIC bifurcation (for which the PRC is well known) then synchrony is stable $[26,50]$. For a splay state of the form $\phi_{i}=i / N$ the eigenvalues of $\widehat{\mathcal{H}}$ are given by $\lambda_{n}=g \sum_{j} H^{\prime}(j / N)\left(\mathrm{e}^{2 \pi i n j / N}-1\right) / N$ 

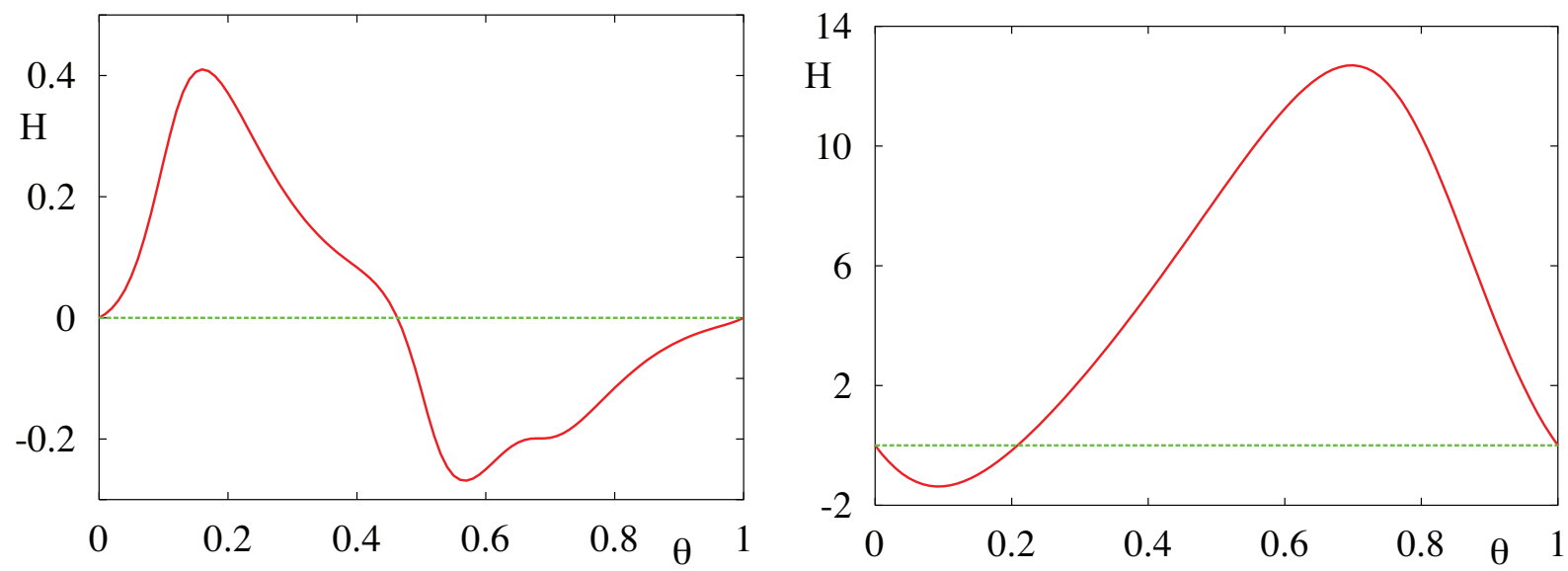

Fig. 4.1. Phase interaction functions corresponding to Fig. 3.4. Left: McKean model. Right: PML model.

for $n=0, \ldots, N-1$. Such solutions are often called merry-go-round states, since all oscillators in the network pass through some fixed phase at regularly spaced time intervals of $T / N$. For a recent review of the stability of cluster states (in which subsets of the oscillator population synchronize, with oscillators belonging to different clusters behaving differently) we refer the reader to $[40,12]$. We shall not focus on them further here.

In the limit $N \rightarrow \infty$ we have the useful result that (for global coupling) network averages may be replaced by time averages:

$$
\lim _{N \rightarrow \infty} \frac{1}{N} \sum_{j=1}^{N} F(j T / N)=\frac{1}{T} \int_{0}^{T} F(t) \mathrm{d} t=F_{0},
$$

for some T-periodic function $F(t)=F(t+T)$. Hence in the large $N$ limit the collective frequency of a splay state (global coupling) is given by $\Omega=1 / T+g H_{0}$, with eigenvalues

$$
\lambda_{n}=\frac{g}{T} \int_{0}^{T} H^{\prime}(t / T) \mathrm{e}^{2 \pi i n t / T} \mathrm{~d} t=-2 \pi i n g H_{-n} .
$$

Hence a splay state is stable if $-n g \operatorname{Im} H_{n}<0$, where we have used the fact that since $H(\theta)$ is real then $\operatorname{Im} H_{-n}=-\operatorname{Im} H_{n}$. A numerical examination of the eigenvalues (4.11) (using the analytical expressions for $H_{n}$ obtained via (4.6)) for the phase interaction functions shown in Fig. 4.1, shows that the splay state is unstable for both these examples. One natural way to stabilize the splay state is to include some synaptic coupling as in the work of $[37,26]$. Another mechanism is to include noise, as originally noted by Kuramoto [57]. If we consider the addition of zero mean white noise with variance $\sigma^{2}$ to the voltage dynamics then the phase-reduced system also feels an additive zero mean white noise source, though with variance $\sigma_{\theta}^{2}$ given by $\sigma_{\theta}^{2}=\sigma^{2} \int_{0}^{T}[R(t)]^{2} \mathrm{~d} t / T$. For a globally coupled network the asynchronous state is stable if $-n g \operatorname{Im} H_{n}<\sigma_{\theta}^{2} n^{2}$, for all $n \neq 0$ [58] . This nicely shows us that if the eigenvalues associated with the deterministic model stray slightly into the right hand complex plane then a small amount of noise can be used to compensate and restabilize the splay state. However, since this is an argument that relies upon weak-coupling then it can necessarily only work if the unstable eigenvalues are sufficiently close to the imaginary axis.

4.2. Beyond weak coupling. Here we develop techniques for the study of the synchronous and splay state in the strong coupling regime for global coupling $\left(g_{i j}=g\right.$, for all $\left.i, j\right)$. First we show how to construct such solutions, extending techniques used in our weak coupling analysis, and use this to explore the effect of gap junction strength on network firing rates. Secondly we show how to analyze the stability of the synchronous solution using Floquet theory, and that of the splay state using a phase-density formalism. 

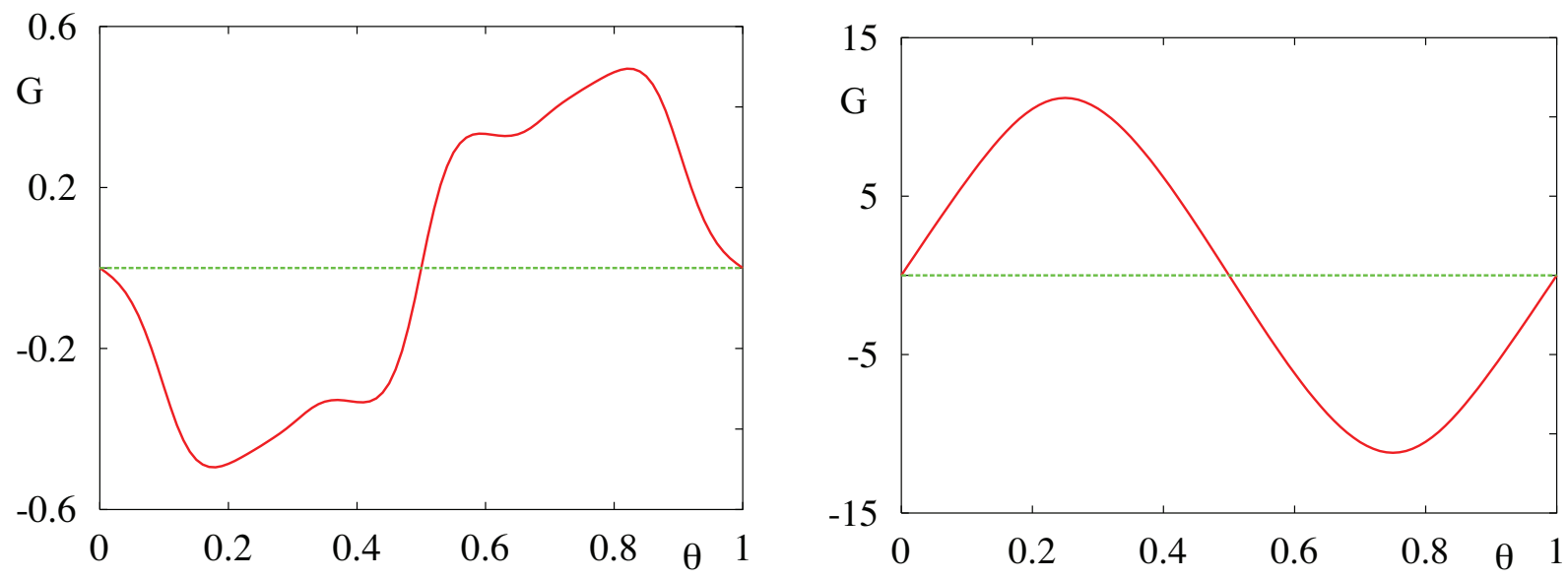

FIG. 4.2. Phase interaction function between two neurons; $G(\theta)=H(-\theta)-H(\theta)$ corresponding to Fig. 4.1. Left: McKean model. Right: PML model.

4.2.1. Existence and stability of a synchronous state. A synchronous network solution exists whenever a periodic orbit for an isolated oscillator exists $(g=0)$, and has the period of the uncoupled isolated oscillator. However, stability will depend on the value of coupling $g$. For convenience we define a matrix $A_{\mu}(g)=A_{\mu}-B(g)$ with

$$
B(g)=\frac{g}{C} J, \quad J=\left[\begin{array}{ll}
1 & 0 \\
0 & 0
\end{array}\right]
$$

Following along similar lines to [40] the $2 N$ Floquet multipliers are given as the eigenvalues of a $2 N \times 2 N$ matrix $G(T)$, where $G(T)$ has the form of equation (3.17), with $D F$ given by

$$
D F=\left[\begin{array}{ccccc}
A(g)+\frac{1}{N} B(g) & \frac{1}{N} B(g) & \frac{1}{N} B(g) & \ldots & \frac{1}{N} B(g) \\
\frac{1}{N} B(g) & A(g)+\frac{1}{N} B(g) & \frac{1}{N} B(g) & \ldots & \frac{1}{N} B(g) \\
\vdots & & \ddots & & \\
\frac{1}{N} B(g) & \frac{1}{N} B(g) & \ldots & A(g)+\frac{1}{N} B(g) & \frac{1}{N} B(g) \\
\frac{1}{N} B(g) & \frac{1}{N} B(g) & \cdots & \frac{1}{N} B(g) & A(g)+\frac{1}{N} B(g)
\end{array}\right],
$$

where $A(g)=A_{\mu}(g)$. Here $\mu$ is chosen according to $\mu=\mu_{1}$ if $t \in\left[0, T_{1}\right), \mu=\mu_{2}$ if $t \in\left[T_{1}, T_{2}\right), \mu=\mu_{3}$ if $t \in\left[T_{2}, T_{3}\right)$ and $\mu=\mu_{4}$ if $t \in\left[T_{3}, T_{4}\right)$, defining four distinct phases of the orbit. On each of these four phases $D F=D F_{\mu}$ is independent of time. The matrix $D F_{\mu}$ is block circulant, with a generating row given by $\left[A_{\mu}(g)+B(g) / N B(g) / N \ldots B(g) / N\right]$, and can be diagonalized by Fourier transform. Introducing the 2 component vector $q_{n}, n=0, \ldots, N-1$, then the components of the eigenvectors of $D F_{\mu}$ can be listed as a set of $N$ 2-component vectors with entries

$$
q_{n} \mathrm{e}^{2 \pi i n m / N}, \quad n, m=0, \ldots, N-1,
$$

where $q_{0}$ is an eigenvector of $A_{\mu}$ and $q_{n \neq 0}$ is an eigenvector of $A_{\mu}(g)$. Hence, we may calculate $G(T)=G_{4}\left(T_{4}\right) G_{3}\left(T_{3}\right) G_{2}\left(T_{2}\right) G_{1}\left(T_{1}\right)$ using the representation $G_{\mu}\left(T_{\mu}\right)=P_{\mu} \exp \left(\Lambda_{\mu} T_{\mu}\right) P_{\mu}^{-1}$, where $P_{\mu}$ is the matrix of eigenvectors of $D F_{\mu}$ and $\Lambda_{\mu}$ the corresponding diagonal matrix of eigenvalues, comprising the 2 eigenvalues of $A_{\mu}$ and $N-1$ copies of the two eigenvalues of $A_{\mu}(g)$. However, motivated by these observations, there is a much simpler way of calculating the Floquet multipliers that avoids the computation of $G(T)$ (and its eigen-structure).

We write the $N$ dimensional linearized system of equations in the form $\dot{Z}=D F Z$. First consider 
the 2 dimensional system $\dot{z}=A(g) z$. By direct inspection we see that

$$
Z=\left[\begin{array}{c}
z \\
-z \\
0 \\
\vdots \\
0
\end{array}\right], \quad\left[\begin{array}{c}
z \\
0 \\
-z \\
\vdots \\
0
\end{array}\right], \ldots,\left[\begin{array}{c}
z \\
0 \\
0 \\
\vdots \\
-z
\end{array}\right]
$$

are linearly independent solutions of $\dot{Z}=D F Z$. Hence the two Floquet multipliers of $\dot{z}=A(\mathrm{~g}) z$ are also Floquet multipliers of $\dot{Z}=D F Z$, with $N-1$ degeneracy. Now consider the 2 dimensional system $\dot{z}=A z$. Again by direct inspection we see that

$$
Z=\left[\begin{array}{c}
z \\
z \\
z \\
\vdots \\
z
\end{array}\right]
$$

is also a solution of $\dot{Z}=D F Z$, with two Floquet multipliers. Hence we can account for all the $2 N$ Floquet multipliers (including the one which is unity). Using the analysis of section 3.2 the three relevant Floquet exponents are given by equation (3.18) and the pair $\left(\sigma_{1}, \sigma_{2}\right)$ where $\sigma_{k}=\ln \left(\mu_{k}\right) / T \bmod 2 \pi i$. Here the $\mu_{k}$ are the two (distinct) eigenvalues of $G(T)=G_{4}\left(T_{4}\right) G_{3}\left(T_{3}\right) G_{2}\left(T_{2}\right) G_{1}\left(T_{1}\right)$, where $G_{\mu}\left(T_{\mu}\right)=\exp \left(A_{\mu}(g) T_{\mu}\right)$ is a $2 \times 2$ matrix. Hence the synchronous network state is stable if an uncoupled isolated oscillator has a stable periodic orbit $(\sigma<0$, and see equation (3.18)), and if the absolute values of the eigenvalues of $G(T)$ are less than unity. The condition for eigenvalues to cross the unit circle along the real axis is $\operatorname{det}[G(T) \pm I]=0$, and off of the real axis we have the condition $\operatorname{det} G(T)=1$. This latter condition is equivalent to $\sum_{\mu} T_{\mu} \operatorname{Tr} A_{\mu}(g)=0$. For the examples in section 4.1 we find that the McKean model supports a stable synchronous state for weak coupling and that this stability persists with increasing $g$. For the PML model the synchronous state is unstable for weak $g$ and can restabilize with increasing $g$ when $\operatorname{det}[G(T)-I]=0$. For the parameters of Fig. 2.2 this occurs at $g \sim 0.45$.

4.2.2. Existence and stability of a splay state. Here we will focus on a globally coupled network in the large $N$ limit. We first re-write the coupling term for a splay state, $\left(v_{i}(t), w_{i}(t)\right)=(v(t-$ $i T / N), w(t-i T / N))$ with $(v(t), w(t))=(v(t+T), w(t+T))$, as

$$
\lim _{N \rightarrow \infty} \frac{1}{N} \sum_{j=1}^{N} v(t+j T / N)=\frac{1}{T} \int_{0}^{T} v(t) \mathrm{d} t
$$

which is independent of both $i$ and $t$. Hence, for a splay state every neuron in the network is described by the same dynamical system, namely

$$
C \dot{v}=f(v)-g v-w+I+g v_{0}, \quad \dot{w}=g(v, w)
$$

where $v_{0}=T^{-1} \int_{0}^{T} v(t) \mathrm{d} t$. We note that because of the dependence on $v_{0}(4.18)$ is an advanced-retarded differential delay equation (see Appendix D for a general numerical method of solution). In the notation of section 3 we write $\dot{z}_{\mu}=A_{\mu}(g) z_{\mu}+b_{\mu}(g)$, where $b_{\mu}(g)=b_{\mu}+b$ with

$$
b=\frac{g}{C} J z_{0}, \quad z_{0}=\left[\begin{array}{c}
v_{0} \\
w_{0}
\end{array}\right] .
$$

The same techniques as deployed in section 3 can be invoked to obtain a formal solution describing a $T$-periodic orbit. In doing so we see that the generalization of (4.6) expresses the solution in terms of 

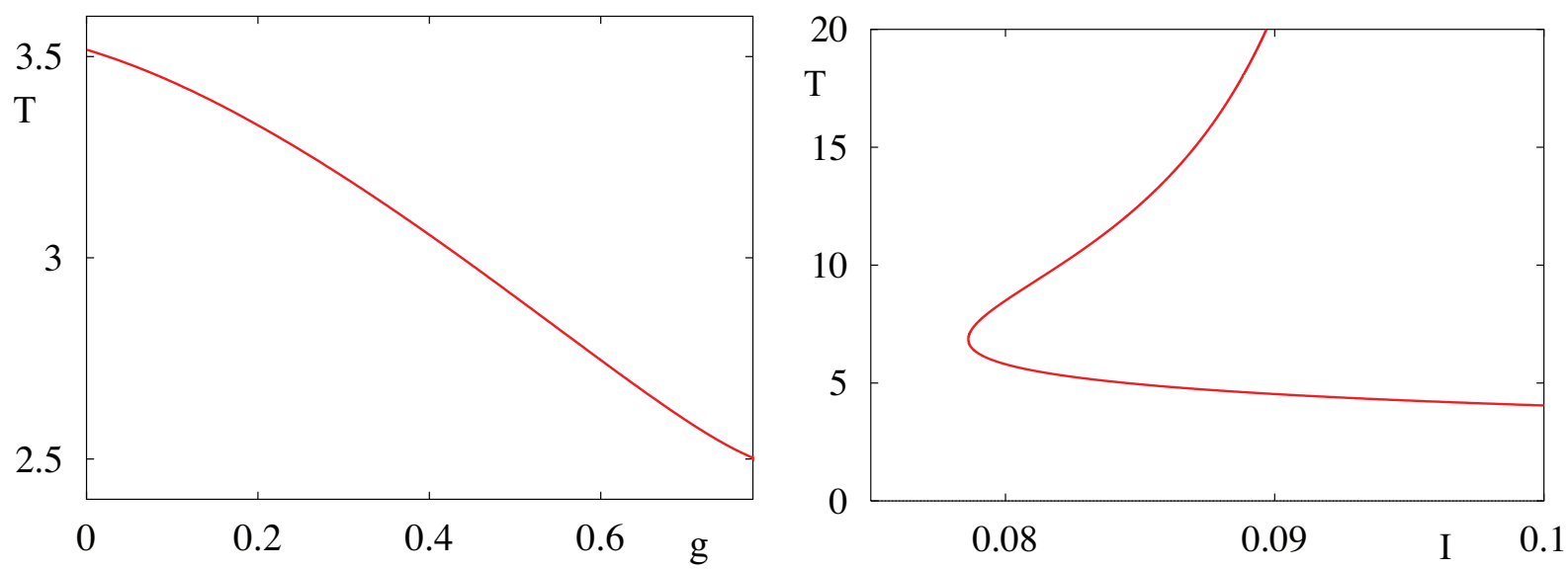

FIG. 4.3. Period of the splay state as a function of the coupling strength $g$. Left: McKean model with parameters as in Fig. 2.1. Right: Period of PML model with $g=0.1$ as a function of drive I and other parameters as in Fig. 2.2. Note the coexistence of a long and short period splay state.

itself via the dependence of $b_{\mu}(g)$ on the Fourier component $z_{0}$. Setting $n=0$ in this equation gives a self consistent expression for $z_{0}$ given by:

$$
z_{0}=\frac{1}{T} \sum_{\nu=1}^{4}\left\{\alpha_{\nu}^{0}(g) z_{\nu}(0)+\gamma_{\nu}^{0}(g) b_{\nu}(g)\right\}
$$

where $\alpha_{\mu}^{n}(g)$ and $\gamma_{\mu}^{n}(g)$ are the natural generalizations of $\alpha_{\mu}^{n}$ and $\gamma_{\mu}^{n}$ (obtained under the replacement of $G_{\mu}(t)=\exp \left(A_{\mu} t\right)$ by $\exp \left(A_{\mu}(g) t\right)$ in (4.7)). Equation (4.20) may be rearranged to obtain an explicit equation for $z_{0}$ in the form $z_{0}=M z_{1}(0)$, where the $2 \times 2$ matrix $M$ is a function of system parameters and the unknowns $w^{*}$ and $T_{\mu}$. The threshold crossing conditions may then be solved for as before to determine $w^{*}$ and $T_{\mu}$. The elements of $\alpha_{\mu}^{0}$ are given explicitly by $K_{\mu}\left(T_{\mu}\right)$ and those of $\gamma_{\mu}^{0}$ are given in Appendix C. The dependence of the period on the strength of coupling $g$ is shown in Fig. 4.3. Typically we find that if a splay state exists for $g=0$ then with increasing $g$ its period decreases. However, in some parameter regimes it can also begin to increase again, as originally noted in [26]. Interestingly for the PML model it is easy to find parameter regimes where there is a coexistence of solutions, as in Fig. 4.3 right. Note that in this example with $I<0.09$ (where there are two solutions) the splay state does not exist at $g=0$ so that weak-coupling theory can not tell us anything about either existence or stability.

In general the stability of a phase-locked state can be determined by determining the $2 N$ Floquet exponents of the linearized system. Indeed pursuing this approach for a splay state we would find a similar coefficient matrix as in (4.13) with diagonal entries not equal to each other, but rather phase shifted, making analytical progress more cumbersome. However for large $N$ we may pursue an alternative phase reduction technique for networks of limit cycle oscillators with synaptic coupling developed by van Vreeswijk [81] and later used to study resonate-and-fire networks [66]. To do this we first write the coupling term $N^{-1} \sum_{j=1}^{N} v_{j}(t)$ in a more convenient form for studying perturbations of the mean field, namely we write

$$
\lim _{N \rightarrow \infty} \frac{1}{N} \sum_{j=1}^{N} v_{j}(t)=\lim _{N \rightarrow \infty} \frac{1}{N} \sum_{j=1}^{N} \sum_{m \in \mathbb{Z}} u\left(t-T_{j}^{m}\right),
$$

where $T_{j}^{m}=m T+j T / N$. Here $u(t)=0$ for $t<0$ and is chosen such that $v(t)=\sum_{m \in \mathbb{Z}} u(t-m T)$, ensuring that $v(t)=v(t+T)$. For arbitrary values of $T_{j}^{m}$ the coupling term (4.21) is time-dependent, and we may write it in the form

$$
E(t)=\int_{0}^{\infty} f(t-s) u(s) \mathrm{d} s, \quad f(t)=\lim _{N \rightarrow \infty} \frac{1}{N} \sum_{j, m} \delta\left(t-T_{j}^{m}\right),
$$


where we recognize $f(t)$ as a firing rate. We now consider perturbations of the mean field such that $E(t)$ (the average membrane voltage) is split into a stationary part (arising from the splay state) and an infinitesimal perturbation. Namely we write $E(t)=v_{0}+\epsilon(t)$, with small $\epsilon(t)$. Since this perturbation to the oscillator defined by (4.18), the splay oscillator, is small we may use phase reduction techniques to study the stability of the splay state.

In terms of a phase $\theta \in[0,1)$ along the asynchronous state we may write the evolution of this phase variable in response to a perturbation in the mean field as

$$
\frac{\mathrm{d} \theta}{\mathrm{d} t}=\frac{1}{T}+g \Gamma(\theta) \epsilon(t)
$$

where $\Gamma(\theta)$ is the ( $g$-dependent) voltage component of the adjoint for the splay oscillator. This can again be calculated in closed form using the techniques developed in section 3.1. Some examples of splay state PRCs are shown in Fig. 4.4. In fact we need to treat $N$ phase variables $\theta_{i}$ each described by an equation
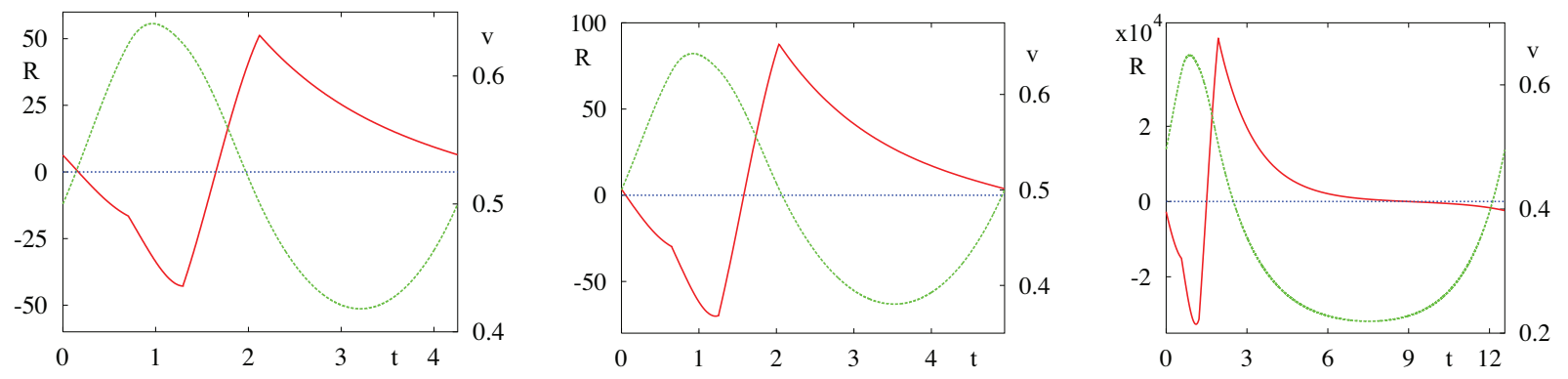

FIG. 4.4. The PRC of the splay state for the PML model for three different points on the solution branch shown in Fig. 4.3 right. Left: $I=0.095$. Middle: $I=0.085$ lower branch. Right: $I=0.085$ upper branch.

of the form (4.23), which are coupled by the dependence of $\epsilon(t)$ on these variables. To make this more explicit we write

$$
\epsilon(t)=\int_{0}^{\infty} \delta f(t-s) u(s) \mathrm{d} s,
$$

and use a phase density description to calculate the dependence of the perturbed firing rate $\delta f$ on the phases. We define a phase density function as the fraction of neurons in the interval $[\theta, \theta+\mathrm{d} \theta]$ namely $\rho(\theta, t)=N^{-1} \sum_{j} \delta\left(\theta_{j}(t)-\theta\right)$. Introducing the flux $J(\theta, t)=\rho(\theta, t) \dot{\theta}$, we have the continuity equation

$$
\frac{\partial \rho}{\partial t}=-\frac{\partial J}{\partial \theta}
$$

with boundary condition $J(1, t)=J(0, t)$. The firing rate is the flux through $\theta=1$, so that $f(t)=J(1, t)$. Considering perturbations around the splay state, $(\rho, J)=\left(1, T^{-1}\right)$, means writing $\rho(\theta, t)=1+\delta \rho(\theta, t)$, with a corresponding perturbation of the flux that takes the form $\delta J(\theta, t)=\delta \rho(\theta, t) / T+g \Gamma(\theta) \epsilon(t)$. In fact the analysis that follows applies to the asynchronous state and not just the splay state. The distinction between the splay and asynchronous states is subtle; in the splay state, the phases are distributed along a cycle with phase differences of $1 / N$ between two adjacent phases. In the asynchronous state, the definition is simply $\rho(\theta, t)=\rho_{0}(\theta)$, namely that the phase density function is independent of time.

Differentiation of $\delta J(\theta, t)$ gives the partial differential equation

$$
\partial_{t} \delta J(\theta, t)=-\frac{1}{T} \partial_{\theta} \delta J(\theta, t)+g \Gamma(\theta) \epsilon^{\prime}(t)
$$

where

$$
\epsilon(t)=\int_{0}^{\infty} u(s) \delta J(1, t-s) \mathrm{d} s .
$$


Assuming a solution of the form $\delta J(\theta, t)=\mathrm{e}^{\lambda t} \delta J(\theta)$, gives

$$
\epsilon(t)=\delta J(1) \mathrm{e}^{\lambda t} \widetilde{u}(\lambda),
$$

where $\widetilde{u}(\lambda)=\int_{0}^{\infty} u(t) \mathrm{e}^{-\lambda t} \mathrm{~d} t$ is the Laplace transform of $u(t)$. In this case $\epsilon^{\prime}(t)=\lambda \epsilon(t)$. Equation (4.26) then reduces to the ordinary differential equation

$$
\frac{\mathrm{d}}{\mathrm{d} \theta} \delta J(\theta) \mathrm{e}^{\lambda T \theta}=g \lambda T \Gamma(\theta) \delta J(1) \widetilde{u}(\lambda) \mathrm{e}^{\lambda T \theta} .
$$

Integrating (4.29) from $\theta=0$ to $\theta=1$ and using the fact that $\delta J(1)=\delta J(0)$ yields an implicit equation for $\lambda$ as

$$
\frac{1}{\widetilde{u}(\lambda)}-\frac{g \lambda T}{\mathrm{e}^{\lambda T}-1} \int_{0}^{1} \Gamma(\theta) \mathrm{e}^{\lambda \theta T} \mathrm{~d} \theta=0
$$

By taking the Laplace transform of $v(t)=\sum_{m} u(t-m T)$, we have that

$$
\widetilde{u}(\lambda)=\left(1-\mathrm{e}^{-\lambda T}\right) \widetilde{v}(\lambda) .
$$

Hence, we may write $\lambda$ as the solution to $\mathcal{E}(\lambda)=0$, where

$$
\mathcal{E}(\lambda)=\frac{\mathrm{e}^{\lambda T}}{\widetilde{v}(\lambda)}-g \lambda T \int_{0}^{1} \Gamma(\theta) \mathrm{e}^{\lambda \theta T} \mathrm{~d} \theta .
$$

Since $1 / \widetilde{v}(0)=0$ we see that $\mathcal{E}(0)=0$ as expected. Writing $\lambda=\nu+i \omega$ then the pair $(\nu, \omega)$ may be found by the simultaneous solution of $\mathcal{E}_{\mathrm{R}}(\nu, \omega)=0$ and $\mathcal{E}_{\mathrm{I}}(\nu, \omega)=0$, where $\mathcal{E}_{\mathrm{R}}(\nu, \omega)=\operatorname{Re} \mathcal{E}(\nu+i \omega)$ and $\mathcal{E}_{\mathrm{I}}(\nu, \omega)=\operatorname{Im} \mathcal{E}(\nu+i \omega)$. In terms of the Fourier coefficients for $\Gamma(\theta)$ and $v(t)$ we may obtain a useful representation for (4.32) using

$$
\begin{aligned}
\int_{0}^{1} \Gamma(\theta) \mathrm{e}^{\lambda \theta T} \mathrm{~d} \theta & =\left(\mathrm{e}^{\lambda T}-1\right) \sum_{n} \frac{R_{n}}{2 \pi i n+\lambda T}, \\
\widetilde{v}(\lambda) & =T \sum_{n} \frac{v_{-n}}{2 \pi i n+\lambda T} .
\end{aligned}
$$

Examples of the spectrum obtained from the zeros of $\mathcal{E}(\lambda)$, for the PML model, are shown in Fig. 4.5. In all cases we find the splay state is unstable.

For small $g$ we expect to recover the stability result obtained using weakly coupled oscillator theory (see section 4.1). To check this we consider solutions of the form $2 \pi i n+\lambda T=2 \pi i n g R_{n} v_{-n} T$, for $n \neq 0$, and $g \ll 1$. In this case we have that

$$
\frac{\mathcal{E}(\lambda)}{g}=\frac{1}{\sum_{n} 1 /\left(2 \pi i n R_{n}\right)}-(\lambda T)^{2} \sum_{n} \frac{R_{n}}{2 \pi i n+\lambda T} .
$$

Using the fact that $R_{n}$ decays as $1 / n$ (and so is an odd function of $n$ ) and $\lambda$ scales with $g$, we may write

$$
\sum_{n, m} \frac{1}{2 \pi i n R_{n}} \frac{R_{m}}{2 \pi i m+\lambda T} \approx \sum_{n} \frac{1}{2 \pi i n(2 \pi i n+\lambda T)}=\frac{1}{\mathrm{e}^{\lambda T}-1} \int_{0}^{1} S(\theta) \mathrm{e}^{\lambda \theta T} d \theta,
$$

where we introduce the function $S(\theta)=\sum_{n} S_{n} \mathrm{e}^{2 \pi i n \theta}$, with $S_{n}=1 /(2 \pi i n)$. Recognizing $S(\theta)$ as the Fourier series for the sawtooth function $S(\theta)=S(\theta+1)$ with $S(\theta)=-\theta$ for $\theta \in[0,1)$, we may evaluate (4.36) as $1 /(\lambda T)^{2}$. Using (4.36) in (4.35) shows that $\mathcal{E}\left(\lambda_{n}\right)=0$, where $\lambda_{n}=2 \pi i n-2 \pi i n g H_{-n}$, and we recover the stability condition for weak coupling, namely $-n g \operatorname{Im} H_{n}<0$, for $n \neq 0$. 

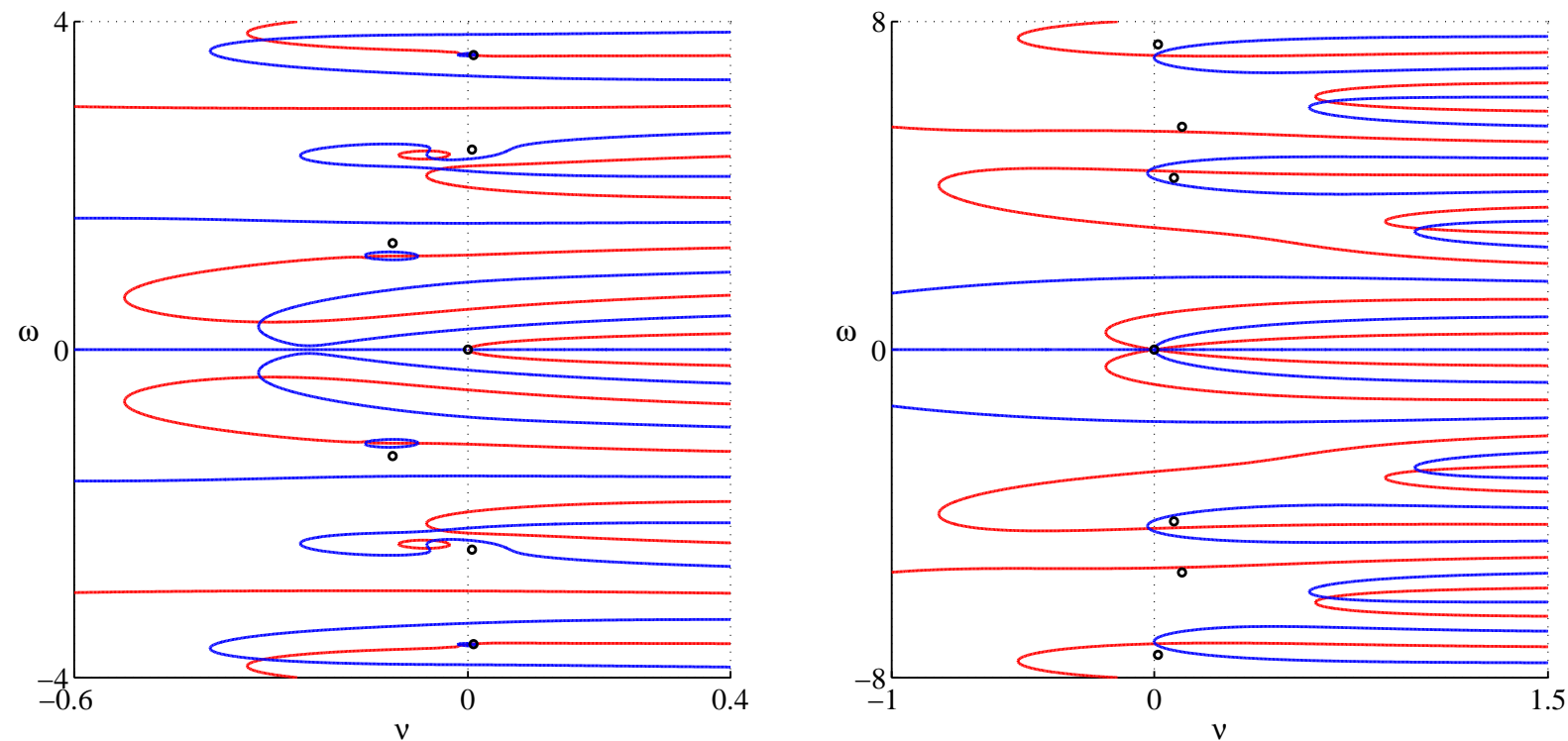

FIG. 4.5. Spectrum for the splay state in the PML model. Eigenvalues are at the positions where the red and blue curves intersect. The small circles denote the predictions from weak coupling theory. Parameters as in Fig. 2.2. Left: $I=0.1, g=0.01$. Note the unstable mode with $\omega \sim \pm 3.6$. As expected eigenvalues from the weak coupling theory are close to the zeros of the full stability function. Right: Spectrum for the splay state with $g=0.1, C=0.9, I=0.085$. In this case predictions from weak coupling theory break down. Note the occurrence of a double-zero eigenvalue signaling a bifurcation to a branch of solutions with $T_{2}=0$ (i.e. orbits tangential to $v=v_{t h}^{2}$ ).

5. Mean field rhythms. We are now in an ideal position to explore observations of Han et al. $[42,43]$ concerning large amplitude oscillations seen in the average membrane potential of globally gap junction coupled Morris-Lecar networks. These novel rhythms were interpreted as cyclic transitions between coherent and incoherent network states, and described as "bursting". However, to distinguish this from the type of behavior commonly associated with fast-slow systems [18] we shall not use this terminology here. For the rest of this section we focus on the PML model. We begin our discussion by analyzing the homogeneous fixed point behavior of the network. Using arguments similar to those in section 4.2.1 we may easily construct conditions for the stability of the fixed point $\left(v^{\mathrm{ss}}, w^{\mathrm{ss}}\right)$. Considering the case $b<v^{\text {ss }}<(1+a) / 2$ then we have that

$$
v^{\mathrm{ss}}=\frac{a-I+b_{s}-b / \gamma_{2}}{1-g-1 / \gamma_{2}}, \quad w^{\mathrm{ss}}=\frac{v^{\mathrm{ss}}}{\gamma_{2}}+b_{s}-\frac{b}{\gamma_{2}} .
$$

If it exists this homogeneous steady state is independent of $C$. The conditions for network stability of this homogeneous state are $\operatorname{Tr} A_{1}=1 / C-1<0$ and $\operatorname{Tr} A_{1}(g)=(1-g) / C-1<0$. Thus a homogeneous network state (if it exists) is only stable for $C>1$, and unstable otherwise. Note however that when $\operatorname{Tr} A_{1}(g)=0$, namely $C=C_{H}=1-g$, we expect the existence of a harmonic splay state (since the dynamics is governed by a purely linear system with imaginary eigenvalues). Generically the results in sections 4.2.1 and 4.2.2 show that both the synchronous and splay states will be unstable for the PML model. However, knowledge of these states and the stability of the network steady state can be used to understand the original observations in [42,43] regarding oscillations in the mean membrane potential. These authors suggested that such states could be viewed as being pushed and pulled between the unstable synchronous state and the unstable fixed point. However, in light of the work presented here we now see that such oscillations may also occur as oscillations around an unstable orbit, that can either be a fixed point or a splay state. We illustrate this idea with the aid of Fig. 5.1. In each of the upper panels we show plots of the unstable orbits that exist for $C<C_{H}, C=C_{H}$ and $C>C_{H}$, with $C<1$. For $C<C_{H}$ there is an unstable splay state that 'sits' between the unstable synchronous state and the unstable homogeneous steady state. Direct numerical simulations show that the network fluctuates 

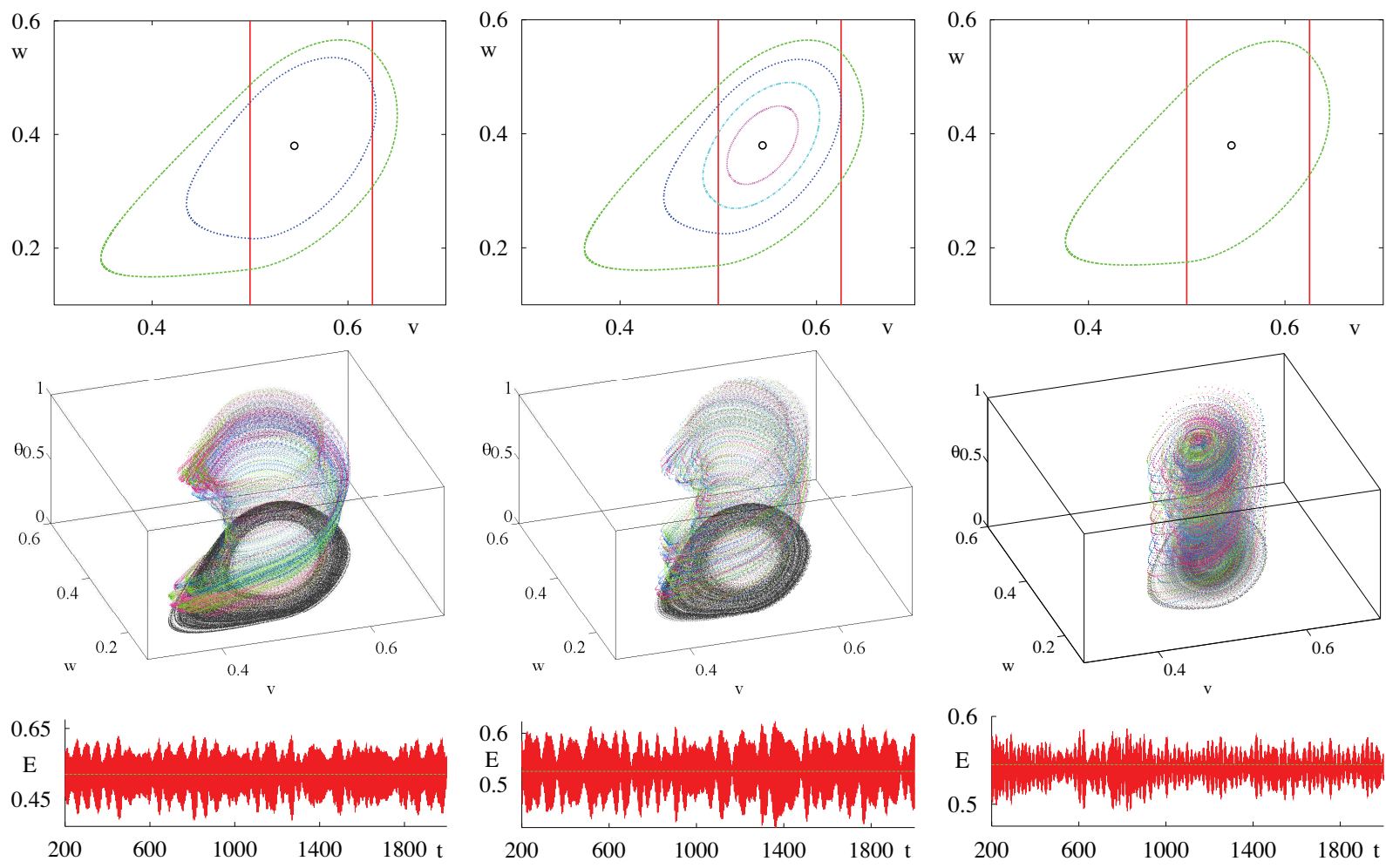

FIG. 5.1. Top: A family of coexisting unstable orbits in the PML model; synchronous (green), splay (blue), subthreshold splay (light blue) and harmonic splay (red). Here $g=0.1, I=0.085$ and other parameters as in Fig. 2.2. left: $C<1-g(C=0.89)$. middle: $C=1-g(C=0.9)$. right: $C>1-g(C=0.91)$. Middle: Numerical simulation (after dropping transients) with $N=100$ neurons showing a pseudo color plot of the triple $\left(\theta, v_{i}, w_{i}\right)$, where $\theta=t / \Delta \bmod 1$ for some fixed $\Delta$. Initial data chosen to lie between the splay and synchronous state. left: The network cycles between the unstable synchronous state and the unstable splay state. $\Delta$ is the chosen as the mean of the synchronous and splay period. middle: The network cycles between the unstable synchronous state and the unstable harmonic splay state. $\Delta$ is the chosen as the mean of the synchronous and harmonic splay period. right: The network cycles between the unstable synchronous state and the unstable fixed point. $\Delta$ is the chosen as the period of the synchronous state. Bottom: Mean field signal E $(t)$ showing large amplitude fluctuations. left: Fluctuations around the splay state (with $\left.v_{0}=0.52107\right)$. middle: Fluctuations around the splay state (with $v_{0}=0.52583$ ). right: Fluctuations around the fixed point (with $\left.v_{0}=0.545\right)$.

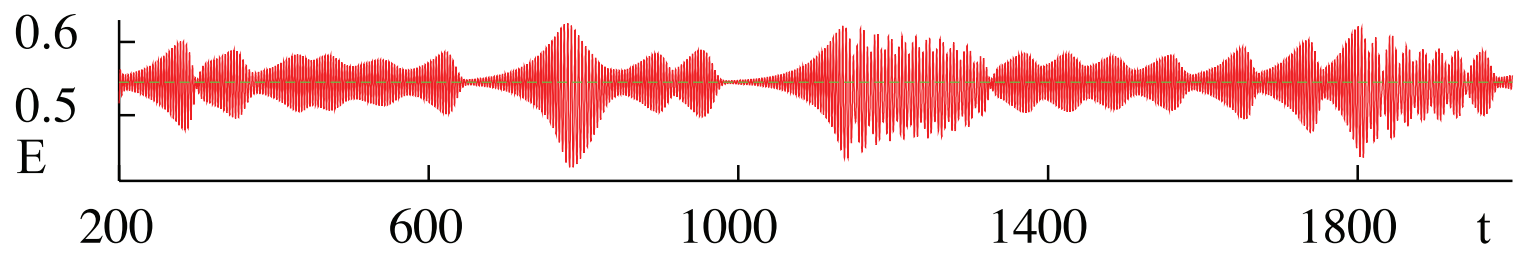

FIG. 5.2. Time varying mean field behavior organized around the unstable fixed point. Parameters as in Fig. 5.1 right with $C=0.95$

around the splay state, cycling between the other two unstable states. A similar behavior occurs at $C=C_{H}$, though the network can fluctuate around and between three co-existing unstable splay states. For $C>C_{H}$ the network dynamics fluctuates around the unstable homogeneous steady state. In Fig. 5.2 we show an example of large amplitude oscillations in the mean membrane potential with a value of $C$ just less than $C=1$, beyond which point the homogeneous steady state is stable.

6. Discussion. Motivated by the desire to understand the dynamics of neuronal networks with gap junction coupling we have developed a number of results for planar piece-wise linear neuron models. We focus on these as they are minimal models capable of generating action potential shapes. Unlike 
synaptically coupled networks the shape of an action potential is all important in a gap junction coupled network as it is communicated directly between cells. For any piece-wise linear planar single neuron model we have shown how to build periodic orbits in a self-consistent way, by piecing together trajectories from neighboring regions of phase space. Moreover, this procedure naturally lends itself to the construction of the associated phase response curve. The stability of periodic orbits has been established using Floquet theory, which in this case generates closed form expressions for the non-zero exponent of the orbit. As well as paving the way for the more obvious weakly coupled network analysis we have found that the simplicity of the piece-wise linear model can allow for studies in the strong coupling regime, albeit for global coupling. In illustration of the utility of studying piece-wise linear networks we have further shown how this can underpin a systematic explanation of the original observations of Han et al. [42] on the generation of exotic mean field signals in networks of Morris-Lecar neurons with gap junction coupling.

Looking forward it is well to mention here a number of possible extensions of the work in this paper that will lead to a deeper understanding of the role of gap junctions in shaping brain rhythms. As we have stressed the techniques in this paper are general and are applicable to many piece-wise linear systems. In particular it would be valuable to study Type I models which rely on a SNIC to generate their firing rate response [33, 32]. The cortical neuron model of Wilson [85] is a classic example of this, and its quadratic recovery variable is easily caricatured by choosing the parameter $\gamma_{1}$ in equation (2.5) to be negative. For systems with local chain-like coupling it may also prove possible to adapt techniques in [65] to study both synchronization and transient dynamics. Another natural step is to endow the purely gap junction coupled networks that we have described here with synaptic interactions. At the level of weak coupling the coupled oscillator theory that we have described here is naturally generalized along the lines in $[26,53]$. Another mechanism available to neurons for the initiation of a firing event is that of anode break excitation, whereby a neuron can fire on release from a hyperpolarized state. The planar models that we have considered here are capable of such behavior, and thus when connected by inhibitory synapses emergent network periodic orbits might also be analyzed in a piece-wise linear fashion. In the strong coupling regime the challenge of studying phase-locked states that are neither synchronous nor splay effectively reduces to the problem of studying ordinary differential equations with delays. The techniques for doing this for piece-wise linear systems are relatively well developed in the engineering community and one may therefore revisit the work in this paper making explicit use of Lambert functions to define trajectories $[6,86]$. In light of the recent interest in the analysis of gap junctions between dendritic trees [73] it would be interesting to explore the possibility of moving away from point neuron models, as studied here, to ones with a spatially extended character [10]. For dendrites without active processes the tools for doing this have been partially developed in [19]. However, of all the possible next steps we regard the development of a tissue level firing rate model that can properly treat gap junction coupling as the major challenge facing the mathematical neuroscience community. Although at the level of fast voltage variables it is natural to think of gap junction coupling between nearest neighbors as generating a diffusive coupling, it is not clear that it is appropriate to simply add diffusive terms to existing rate models [77], such as the Wilson-Cowan, Amari or Liley models (recently reviewed in [20]), since the state variables in these examples have no direct interpretation as fast voltage variables. In future work we hope to combine ideas from mean field dynamics, particularly those in [51,67], with "equation-free" modeling [59] to tackle this challenge.

Acknowledgments. I would like to thank Bard Ermentrout and three anonymous referees for useful comments that have improved the presentation of the work in this paper. 
Appendix A. For real eigenvalues of $A$, we have the explicit form for $G(t)$ in section 3 :

$$
\begin{aligned}
& G_{11}(t)=\frac{1}{\lambda_{+}-\lambda_{-}}\left\{\lambda_{+} \mathrm{e}^{\lambda_{+} t}-\lambda_{-} \mathrm{e}^{\lambda_{-} t}-a_{22}\left[\mathrm{e}^{\lambda_{+} t}-\mathrm{e}^{\lambda_{-} t}\right]\right\}, \\
& G_{12}(t)=-\frac{\lambda_{+}-a_{22}}{\lambda_{+}-\lambda_{-}} \frac{\lambda_{-}-a_{22}}{a_{21}}\left[\mathrm{e}^{\lambda_{+} t}-\mathrm{e}^{\lambda_{-} t}\right], \\
& G_{21}(t)=\frac{a_{21}}{\lambda_{+}-\lambda_{-}}\left[\mathrm{e}^{\lambda_{+} t}-\mathrm{e}^{\lambda_{-}}\right], \\
& G_{22}(t)=\frac{1}{\lambda_{+}-\lambda_{-}}\left\{\lambda_{+} \mathrm{e}^{\lambda_{-} t}-\lambda_{-} \mathrm{e}^{\lambda_{+} t}+a_{22}\left[\mathrm{e}^{\lambda_{+} t}-\mathrm{e}^{\lambda_{-} t}\right]\right\} .
\end{aligned}
$$

The matrix $K(t)$ may then be calculated as:

$$
\begin{aligned}
& K_{11}(t)=\frac{1}{\lambda_{+}-\lambda_{-}}\left\{\mathrm{e}^{\lambda_{+} t}-\mathrm{e}^{\lambda_{-} t}-a_{22}\left[\frac{\mathrm{e}^{\lambda_{+} t}-1}{\lambda_{+}}-\frac{\mathrm{e}^{\lambda_{-} t}-1}{\lambda_{-}}\right]\right\}, \\
& K_{12}(t)=-\frac{\lambda_{+}-a_{22}}{\lambda_{+}-\lambda_{-}} \frac{\lambda_{-}-a_{22}}{a_{21}}\left[\frac{\mathrm{e}^{\lambda_{+} t}-1}{\lambda_{+}}-\frac{\mathrm{e}^{\lambda_{-} t}-1}{\lambda_{-}}\right], \\
& K_{21}(t)=\frac{a_{21}}{\lambda_{+}-\lambda_{-}}\left[\frac{\mathrm{e}^{\lambda_{+} t}-1}{\lambda_{+}}-\frac{\mathrm{e}^{\lambda_{-} t}-1}{\lambda_{-}}\right], \\
& K_{22}(t)=\frac{1}{\lambda_{+}-\lambda_{-}}\left\{\frac{\lambda_{+}}{\lambda_{-}}\left[\mathrm{e}^{\lambda_{-} t}-1\right]-\frac{\lambda_{-}}{\lambda_{+}}\left[\mathrm{e}^{\lambda_{+} t}-1\right]+a_{22}\left[\frac{\mathrm{e}^{\lambda_{+} t}-1}{\lambda_{+}}-\frac{\mathrm{e}^{\lambda_{-} t}-1}{\lambda_{-}}\right]\right\} .
\end{aligned}
$$

For complex eigenvalues of $A$, we have the explicit form for $G(t)$ :

$$
G(t)=\frac{\mathrm{e}^{\rho t}}{\widehat{\omega}}\left[\begin{array}{cc}
\widehat{\omega} \cos \omega t-\widehat{\rho} \sin \omega t & \sin \omega t \\
-\left(\widehat{\rho}^{2}+\widehat{\omega}^{2}\right) \sin \omega t & \widehat{\omega} \cos \omega t+\widehat{\rho} \sin \omega t
\end{array}\right] .
$$

The matrix $K(t)$ may then be calculated as:

$$
K(t)=\frac{1}{\widehat{\omega}}\left[\begin{array}{cc}
\widehat{\omega} K_{R}(t)-\widehat{\rho} K_{I}(t) & K_{I}(t) \\
-\left(\widehat{\rho}^{2}+\widehat{\omega}^{2}\right) K_{I}(t) & \widehat{\omega} K_{R}(t)+\widehat{\rho} K_{I}(t)
\end{array}\right]
$$

where

$$
\begin{aligned}
K_{R}(t) & =\frac{1}{\rho^{2}+\omega^{2}}\left\{\rho\left[\mathrm{e}^{\rho t} \cos (\omega t)-1\right]+\omega \mathrm{e}^{\rho t} \sin (\omega t)\right\}, \\
K_{I}(t) & =\frac{1}{\rho^{2}+\omega^{2}}\left\{\omega\left[1-\mathrm{e}^{\rho t} \cos (\omega t)\right]+\rho \mathrm{e}^{\rho t} \sin (\omega t)\right\} .
\end{aligned}
$$

Appendix B. Computationally useful forms for the matrix elements in (4.7) are as follows:

$$
\begin{aligned}
\alpha_{\mu}^{n} & =P_{\mu} \Psi_{\mu}^{n} P_{\mu}^{-1}, \\
\beta_{\mu}^{n} & =\widetilde{P}_{\mu} \Psi_{\mu}^{-n} \widetilde{P}_{\mu}^{-1} \mathrm{e}^{-2 \pi i n T_{\mu} / T}, \\
\gamma_{\mu}^{n} & =P_{\mu} \widetilde{\Psi}_{\mu}^{n} P_{\mu}^{-1},
\end{aligned}
$$

where

$$
\begin{aligned}
& \Psi_{\mu}^{n}=\operatorname{diag}\left(\frac{\mathrm{e}^{\left(\lambda_{+}^{\mu}-2 \pi i n / T\right) T_{\mu}}-1}{\lambda_{+}^{\mu}-2 \pi i n / T}, \frac{\mathrm{e}^{\left(\lambda_{-}^{\mu}-2 \pi i n / T\right) T_{\mu}}-1}{\lambda_{-}^{\mu}-2 \pi i n / T}\right), \\
& \widetilde{\Psi}_{\mu}^{n}=\operatorname{diag}\left(1 / \lambda_{+}^{\mu}, 1 / \lambda_{-}^{\mu}\right)\left[\Psi_{\mu}^{n}+\frac{\mathrm{e}^{-2 \pi i n T_{\mu} / T}-1}{2 \pi i n / T} I\right], \quad n \neq 0, \\
& \widetilde{\Psi}_{\mu}^{0}=\operatorname{diag}\left(1 / \lambda_{+}^{\mu}, 1 / \lambda_{-}^{\mu}\right) \operatorname{diag}\left(\frac{\mathrm{e}^{\lambda_{+}^{\mu} T_{\mu}}-1}{\lambda_{+}^{\mu}}-T_{\mu}, \frac{\mathrm{e}^{\lambda_{-}^{\mu} T_{\mu}}-1}{\lambda_{-}^{\mu}}-T_{\mu}\right),
\end{aligned}
$$

with $P_{\mu}$ and $\widetilde{P}_{\mu}$ as the matrix of eigenvectors of $A_{\mu}$ and $A_{\mu}^{T}$ respectively, with associated eigenvalues $\lambda_{ \pm}^{\mu}$. From the structure of $\Psi_{\mu}^{n}$ and $\widetilde{\Psi}_{\mu}^{n}$ above we see that $\alpha_{\mu}^{n}, \beta_{\mu}^{n}$, and $\gamma_{\mu}^{n}$ all decrease as $1 / n$. 
Appendix C. In section 4.2.2 the elements of $\gamma_{\mu}^{0}$ are calculated explicitly by noting that $\gamma_{\mu}^{0}=$ $\int_{0}^{T_{\mu}} K_{\mu}(t) \mathrm{d} t$. The structure of $K_{\mu}(t)$ for real and imaginary eigenvalues of the associated matrix $A_{\mu}$ are given by (6.2) and (6.4) respectively. Denoting $\int_{0}^{t} K(s) \mathrm{d} s$ by $F(t)$ gives

$$
\begin{aligned}
F_{11}(t) & =\frac{1}{\lambda_{+}-\lambda_{-}}\left\{\frac{\mathrm{e}^{\lambda_{+} t}-1}{\lambda_{+}}-\frac{\mathrm{e}^{\lambda_{-} t}-1}{\lambda_{-}}-a_{22}\left[\frac{1}{\lambda_{+}}\left[\frac{\mathrm{e}^{\lambda_{+} t}-1}{\lambda_{+}}-t\right]-\frac{1}{\lambda_{-}}\left[\frac{\mathrm{e}^{\lambda_{-} t}-1}{\lambda_{-}}-t\right]\right]\right\}, \\
F_{12}(t) & =-\frac{\lambda_{+}-a_{22}}{\lambda_{+}-\lambda_{-}} \frac{\lambda_{-}-a_{22}}{a_{21}}\left[\frac{1}{\lambda_{+}}\left[\frac{\mathrm{e}^{\lambda_{+} t}-1}{\lambda_{+}}-t\right]-\frac{1}{\lambda_{-}}\left[\frac{\mathrm{e}^{\lambda_{-} t}-1}{\lambda_{-}}-t\right]\right], \\
F_{21}(t) & =\frac{a_{21}}{\lambda_{+}-\lambda_{-}}\left[\frac{1}{\lambda_{+}}\left[\frac{\mathrm{e}^{\lambda_{+} t}-1}{\lambda_{+}}-t\right]-\frac{1}{\lambda_{-}}\left[\frac{\mathrm{e}^{\lambda_{-} t}-1}{\lambda_{-}}-t\right]\right], \\
F_{22}(t) & =\frac{1}{\lambda_{+}-\lambda_{-}}\left\{\frac{\lambda_{+}}{\lambda_{-}}\left[\frac{\mathrm{e}^{\lambda_{-} t}-1}{\lambda_{-}}-t\right]-\frac{\lambda_{-}}{\lambda_{+}}\left[\frac{\mathrm{e}^{\lambda_{+} t}-1}{\lambda_{+}}-t\right]\right. \\
& \left.+a_{22}\left[\frac{1}{\lambda_{+}}\left[\frac{\mathrm{e}^{\lambda_{+}}-1}{\lambda_{+}}-t\right]-\frac{1}{\lambda_{-}}\left[\frac{\mathrm{e}^{\lambda_{-}}-1}{\lambda_{-}}-t\right]\right]\right\} .
\end{aligned}
$$

for real $\lambda_{ \pm}$and

$$
F(t)=\frac{1}{\widehat{\omega}}\left[\begin{array}{cc}
\widehat{\omega} F_{R}(t)-\widehat{\rho} F_{I}(t) & F_{I}(t) \\
-\left(\widehat{\rho}^{2}+\widehat{\omega}^{2}\right) F_{I}(t) & \widehat{\omega} F_{R}(t)+\widehat{\rho} F_{I}(t)
\end{array}\right]
$$

where

$$
\begin{aligned}
& F_{R}(t)=\frac{1}{\rho^{2}+\omega^{2}}\left\{\rho\left[K_{R}(t)-t\right]+\omega K_{I}(t)\right\}, \\
& F_{I}(t)=\frac{1}{\rho^{2}+\omega^{2}}\left\{\omega\left[t-K_{R}(t)\right]+\rho K_{I}(t)\right\},
\end{aligned}
$$

for complex $\lambda_{ \pm}$. The matrices $\gamma_{\mu}^{0}=F_{\mu}\left(T_{\mu}\right)$ may then be calculated using the above forms for $F(t)$ (under the replacement of $\lambda_{ \pm}$by $\lambda_{ \pm}^{\mu}$ ).

Appendix D. For a splay state we may rewrite the advanced-retarded system of equations (4.18) as a set of ODEs by introducing:

$$
X_{-}(t)=\frac{1}{T} \int_{0}^{t} v(t) \mathrm{d} t, \quad X_{+}(t)=\frac{1}{T} \int_{t}^{T} v(t) \mathrm{d} t .
$$

After re-scaling time as $\tau=t / T$ we may write

$$
\begin{aligned}
\frac{C}{T} \frac{\mathrm{d} v}{\mathrm{~d} \tau} & =f(v)-g v-w+I+g\left(X_{-}+X_{+}\right), \\
\frac{1}{T} \frac{\mathrm{d} w}{\mathrm{~d} \tau} & =g(v, w), \\
\frac{\mathrm{d} X_{-}}{\mathrm{d} \tau} & =v \\
\frac{\mathrm{d} X_{+}}{\mathrm{d} \tau} & =-v,
\end{aligned}
$$

subject to the boundary conditions $v(0)=v_{\text {th }}=v(1), w(0)=w^{*}=w(1), X_{-}(0)=0, X_{-}(1)=v_{0}$, $X_{+}(0)=v_{0}$, and $X_{+}(1)=0$ (for some voltage section $v_{\text {th }}$ ). We have four ODEs with seven boundary conditions which we may treat as a boundary value problem for the free parameters $\left(v_{0}, w^{*}, T\right)$. For general choices of $f$ and $g$ it is natural to use numerical shooting for the solution of this problem. Alternatively, for the PWL models discussed in this paper we may analytically construct solutions according to the prescription described in section 4.2.2. 


\section{REFERENCES}

[1] L. F. Abbott and T. B. Kepler, Model neurons: from Hodgkin-Huxley to Hopfield, in Statistical Mechanics of Neural Networks, L. Garrido, ed., no. 368 in Lecture notes in Physics, Springer-Verlag, 1990, pp. 5-18.

[2] D. J. Allwright, Harmonic balance and the Hopf bifurcation, Mathematical Proceedings of the Cambridge Philosophical Society, 82 (1977), pp. 453-467.

3] A. V. Alvarez, C. C. Chow, E. J. V. Bockstaele, and J. T. Williams, Frequency-dependent synchrony in locus ceruleus: Role of electrotonic coupling, Proceedings of the National Academy of Sciences, 99 (2002), pp. 40324036.

[4] Y. Amitai, J. R. Gibson, M. B. S. L. Patrick, A. M. Ho, B. W. Connors, and D. Golomb, The spatial dimensions of electrically coupled networks of interneurons in the neocortex, Journal of Neuroscience, 22 (2002), pp. 41424152 .

[5] P. Ashwin And J. W. Swift, The dynamics of $n$ weakly coupled identical oscillators, Journal of Nonlinear Science, 2 (1992), pp. 69-108.

[6] F. A. Asl And A. G. Ulsoy, Analysis of a system of linear delay differential equations, Journal of Dynamic Systems, Measurement, and Control, 125 (2003), pp. 215-223.

[7] T. Bem And J. Rinzel, Short duty cycle destabilizes a half-center oscillator, but gap junctions can restabilize, Journal of Neurophysiology, 91 (2003), pp. 693-703.

[8] M. V. L. BENNET AND R. S. Zukin, Electrical coupling and neuronal synchronization in the mammalian brain, Neuron, 41 (2004), pp. 495-511.

[9] M. Bennett, J. Contreras, F. Bukauskas, And J. SÁez, New roles for astrocytes: gap junction hemichannels have something to communicate, Trends in Neurosciences, 26 (2003), pp. 610-617.

[10] P. C. Bressloff And S. Coombes, Physics of the extended neuron, International Journal of Modern Physics B, 11 (1997), pp. 2343-2392.

11] — Dynamics of strongly coupled spiking neurons, Neural Computation, 12 (2000), pp. 91-129.

[12] E. Brown, P. Holmes, And J. Moehlis, Perspectives and Problems in Nonlinear Science: A Celebratory Volume in Honor of Larry Sirovich, Springer, New York, 2003, ch. Globally coupled oscillator networks, pp. 183-215.

[13] E. Brown, J. Moenlis, AND P. Holmes, On the phase reduction and response dynamics of neural oscillator populations, Neural Computation, 16 (2004), pp. 673-715.

[14] C. Chicone, Ordinary differential equations with applications, vol. 34 of Texts in Applied Mathematics, Springer, second ed., 2006.

[15] C. C. Chow And N. Kopell, Dynamics of spiking neurons with electrical coupling., Neural Computation, 12 (2000), pp. $1643-78$.

[16] B. W. Connors And M. A. Long, Electrical synapses in the mammalian brain, Annual Review of Neuroscience, 27 (2004), pp. 393-418.

[17] S. Coombes, Phase locking in networks of synaptically coupled McKean relaxation oscillators, Physica D, 160 (2001), pp. $173-188$.

[18] S. Coombes and P. C. Bressloff, eds., Bursting: The Genesis of Rhythm in the Nervous System, World Scientific Press, 2005.

[19] S. Coombes, Y. Timofeeva, C.-M. Svensson, G. J. Lord, K. Josic, S. J. Cox, and C. M. Colbert, Branching dendrites with resonant membrane: A "sum-over-trips" approach, Biological Cybernetics, 97 (2007), pp. 137-149.

[20] S. Coombes, N. A. Venkov, L. Shiau, I. Bojak, D. T. J. Liley, and C. R. Laing, Modeling electrocortical activity through improved local approximations of integral neural field equations, Physical Review E, 76 (2007), pp. 051901(1-8).

[21] G. De Vries and A. Sherman, Bursting: The Genesis of Rhythm in the Nervous System, World Scientific, 2005, ch. Beyond synchronization: modulatory and emergent effects of coupling in square-wave bursting.

[22] M. Denman-Johnson And S. Coombes, A continuum of weakly coupled McKean neurons, Physical Review E, 67 (2003), p. 051903.

[23] S. Dhein AND J. S. Borer, eds., Cardiovascular Gap Junctions (Advances in Cardiology), S Karger AG, 2006.

[24] F. E. DudeK, Gap junctions and fast oscillations: A role in seizures and epileptogenesis?, Epilepsy Currents, 2 (2002), pp. 133-136.

[25] B. Ermentrout, Simulating, Analyzing, and Animating Dynamical Systems: A Guide to Xppaut for Researchers and Students, Society for Industrial \& Applied Mathematics, 2002.

[26] - Gap junctions destroy persistent states in excitatory networks, Physical Review E, 74 (2006), pp. 031918(1-8).

[27] G. B. Ermentrout, Neural nets as spatio-temporal pattern forming systems, Reports on Progress in Physics, 61 (1998), pp. 353-430

[28] G. B. Ermentrout And N. Kopell, Frequency plateaus in a chain of weakly coupled oscillators, SIAM Journal on Applied Mathematics, 15 (1984), pp. 215-237.

[29] - Oscillator death in systems of coupled neural oscillators, SIAM Journal on Applied Mathematics, 50 (1990), pp. 125-146.

[30] - Multiple pulse interactions and averaging in systems of coupled neural oscillators, Journal of Mathematical Biology, 29 (1991), pp. 195-217.

[31] R. Fitzhugh, Impulses and physiological states in theoretical models of nerve membranes, Biophysical Journal, 1182 (1961), pp. 445-466.

[32] H. Fujil AND I. Tsuda, Itinerant dynamics of class $I^{*}$ neurons coupled by gap junctions, in Computational Neuroscience: Cortical Dynamics, P. Érdi, A. Esposito, M. Marinaro, and Scarpetta, eds., Lecture Notes in Computer 
Science, Springer-Verlag, 2004, pp. 140-160.

[33] — Neocortical gap junction-coupled interneuron systems may induce chaotic behavior itinerant among quasiattractors exhibiting transient synchrony, Neurocomputing, 58-60 (2004), pp. 151-157.

[34] T. Fukuda, T. Kosaka, W. Singer, and R. A. W. Galuske, Gap junctions among dendrites of cortical GABAergic neurons establish a dense and widespread intercolumnar network, The Journal of Neuroscience, 26 (2006), pp. 3434-3443.

[35] E. J. Furshpan And D. D. Potter, Mechanism of nerve-impulse transmission at a crayfish synapse, Nature, 180 (1957), pp. 342-343.

[36] M. Galarreta And S. Hestrin, A network of fast-spiking cells in the neocortex connected by electrical synapses, Nature, 402 (1999), pp. 72-75.

[37] J. GaO And P. Holmes, On the dynamics of electrically-coupled neurons with inhibitory synapses, Journal of Computational Neuroscience, 22 (2007), pp. 39-61.

[38] W. Gerstner and J. L. van Hemmen, Associative memory in a network of 'spiking' neurons, Network, 3 (1992), pp. 139-164.

[39] J. R. Gibson, M. Beierlein, And B. W. Connors, Functional properties of electrical synapses between inhibitory interneurons of neocortical layer 4, Journal of Neurophysiology, 93 (2005), pp. 467-480.

[40] D. Golomb, X. J. Wang, And J. Rinzel, Synchronization properties of spindle oscillations in a thalamic reticular nucleus model, Journal of Neurophysiology, 72 (1994), pp. 1109-1126.

[41] F. Hamzei-Sichani, N. Kamasama, W. G. M. Janssen, T. Yasumura, K. G. V. Davidson, P. R. Hof, S. L. wearne, M. G. Stewart, S. R. young, M. A. Whittington, J. E. Rash, and R. D. Traub, Gap junctions on hippocampal mossy fiber axons demonstrated by thin-section electron microscopy and freeze-fracture replica immunogold labeling, Proceedings of the National Acadamy of Sciences USA, 104 (2007), pp. 12548-12553.

[42] S. K. Han, C. Kurrer, and Y. Kuramoto, Dephasing and bursting in coupled neural oscillators, Physical Review Letters, 75 (1995), pp. 3190-3193.

[43] — Diffusive interaction leading to dephasing of coupled neural oscillators, International Journal of Bifurcation and Chaos, 7 (1997), pp. 869-876.

[44] A. L. Hodgkin And A. F. Huxley, A quantitative description of membrane current and its application to conduction and excitation in nerve tissue, Journal of Physiology (London), 116 (1952), pp. 449-472.

[45] F. C. Hoppensteadt and E. M. Izhikevich, Weakly Connected Neural Networks, no. 126 in Applied Mathematical Sciences, Springer-Verlag, New York, 1997.

[46] S. G. Hormuzdi, M. A. Filippov, G. Mitropoulou, H. Monyer, and R. Bruzzone, Electrical synapses: a dynamic signaling system that shapes the activity of neuronal networks, Biochimica et Biophysica Acta, 1662 (2004), pp. 113-137.

[47] S. W. Hughes And V. CRuneldi, Just a phase they're going through: the complex interaction of intrinsic highthreshod bursting and gap junctions in the generation of thalamic $\alpha$ and $\theta$ rhythms, International Journal of Psychophysiology, 74 (2007), pp. 3-17.

[48] E. M. IzhikeVich, Phase equations for relaxation oscillators, SIAM Journal on Applied Mathematics, 60 (2000), pp. 1789-1805.

[49] - Dynamical Systems in Neuroscience: The Geometry of Excitability and Bursting, The MIT Press, 2007.

[50] _ Dynamical Systems in Neuroscience: The Geometry of Excitability and Bursting, The MIT Press, 2007, ch. 10. Synchronization (available online at www.izhikevich.com).

[51] C. G. A. V. K. JiRsa AND J. A. S. Kelso, Synchrony and clustering in heterogeneous networks with global coupling and parameter dispersion, Physical Review Letters, 94 (2005), p. 018106.

[52] J. Karbowski AND N. KopelL, Multispikes and synchronization in a large-scale neural network with delays, Neural Computation, 12 (2000), pp. 1573-1606.

[53] F. G. Kazanci And B. Ermentrout, Pattern formation in an array of oscillators with electrical and chemical coupling, SIAM Journal on Applied Mathematics, 67 (2007), pp. 512-529.

[54] T. B. Kepler, L. F. AbBott, And E. Marder, Reduction of conductance-based neuron models, Biological Cybernetics, 66 (1992), pp. 381-387.

[55] T. B. Kepler, E. Marder, And L. F. Аввоtt, The effect of electrical coupling on the frequency of model neuronal oscillators, Science, 248 (1990), pp. 83-85.

[56] N. Kopell and B. Ermentrout, Chemical and electrical synapses perform complementary roles in the synchronization of interneuronal networks, Proceedings of the National Acadamy of Sciences USA, 101 (2004), pp. 1548215487.

[57] Y. Kuramoto, Chemical Oscillations, Waves and Turbulence, Springer-Verlag, 1984.

[58] — Collective synchronization of pulse-coupled oscillators and excitable units, Physica D, 50 (1991), pp. 15-30.

[59] C. R. LAING, On the application of "equation-free modelling" to neural systems, Journal of Computational Neuroscience, 20 (2006), pp. 5-23.

[60] T. J. Lewis AND J. Rinzel, Self-organized synchronous oscillations in a network of excitable cells coupled by gap junctions, Network, 11 (2000), pp. 299-320.

[61] — Dynamics of spiking neurons connected by both inhibitory and electrical coupling, Journal of Computational Neuroscience, 14 (2003), pp. 283-309.

[62] Y. Loewenstein, Y. YAROM, And H. Sompolinsky, The generation of oscillations in networks of electrically coupled cells, Proceedings of the National Acadamy of Sciences USA, 98 (2001), pp. 8095-8100.

[63] J. G. Mancilla, T. J. Lewis, D. J. Pinto, J. Rinzel, and B. W. Connors, Synchronization of electrically coupled pairs of inhibitory interneurons in neocortex, The Journal of Neuroscience, 27 (2007), pp. 2058-2073.

[64] H. P. McKean, Nagumo's equation, Advances in Mathematics, 4 (1970), pp. 209-223. 
[65] G. S. Medvedev And N. Kopell, Synchronization and transient dynamics in the chains of electrically coupled FitzHugh-Nagumo oscillators, SIAM Journal on Applied Mathematics, 61 (2001), pp. 1762-1801.

[66] K. Miura And M. Okada, Globally coupled resonate-and-fire models, Progress of Theoretical Physics Supplement, 161 (2006), pp. 255-259.

[67] S. D. Monte, F. D’Ovidio, And E. Mosekilde, Coherent regimes of globally coupled dynamical systems, Physical Review Letters, 90 (2003), pp. 054102(1-4).

[68] C. Morris And H. Lecar, Voltage oscillations in the barnacle giant muscle fiber, Biophysical Journal, 35 (1981), pp. 193-213.

[69] B. Pfeuty, G. Mato, D. Golomb, and D. Hansel, Electrical synapses and synchrony: the role of intrinsic currents, The Journal of Neuroscience, 23 (2003), pp. 6280-6294.

[70] — The combined effects of inhibitory and electrical synapses in synchrony, Neural Computation, 17 (2005), pp. 633-670.

[71] J. E. Rash, R. K. Dillman, B. L. Bilhartz, H. S. Duffy, L. R. Whalen, and T. Yasumura, Mixed synapses discovered and mapped throughout mammalian spinal cord, Proceedings of the National Acadamy of Sciences USA, 93 (1996), pp. 4235-4239.

[72] J. Rubin And D. TeRman, Geometric singular perturbation analysis of neuronal dynamics, in Handbook of Dynamical Systems, B. Fiedler, G. Ioos, and N. Kopell, eds., vol. 2, Elsevier,, 2002.

[73] F. Saraga, L. NG, AND F. K. Skinner, Distal gap junctions and active dendrites can tune network dynamics, Journal of Neurophysiology, 95 (2006), pp. 1669-1682.

[74] A. Sherman And J. Rinzel, Rhythmogenic effects of weak electrotonic coupling in neuronal models, Proceedings of the National Acadamy of Sciences USA, 89 (1992), pp. 2471-2474.

[75] C. Sotelo, R. Llinas, AND R. BAKeR, Structural study of inferior olivary nucleus of the cat: morphological correlates of electrotonic coupling, Journal of Neurophysiology, 37 (1974), pp. 541-559.

[76] C. Soto-Treviño, P. Rabbah, E. Marder, and F. Nadim, Computational model of electrically coupled, intrinsically distinct pacemaker neurons, Journal of Neurophysiology, 94 (2005), pp. 590-604.

[77] M. L. Steyn-Ross, D. A. Steyn-Ross, M. T. Wilson, and J. W. Sleigh, Gap junctions mediate large-scale Turing structures in a mean-field cortex driven by subcortical noise, Physical Review E, 76 (2007), pp. 011916(1-16).

[78] A. Tonnelier, The McKean's caricature of the FitzHugh-Nagumo model I. the space-clamped system, SIAM Journal on Applied Mathematics, 63 (2002), pp. 459-484.

[79] —, McKean model, Scholarpedia, (2007), p. 12071.

[80] A. Tonnelier And W. Gerstner, Piecewise linear differential equations and integrate-and-fire neurons: insights from two-dimensional membrane models, Physical Review E, 67 (2003), pp. 021908(1-16).

[81] C. VAn VReEswiJk, Analysis of the asynchronous state in networks of strongly coupled oscillators, Physical Review Letters, 84 (2000), pp. 5110-5113.

[82] J. L. P. VelazQuez And P. L. Carlen, Gap junctions, synchrony and seizures, Trends in Neurosciences, 23 (2000), pp. 68-74.

[83] W. P. WANG, Multiple impulse solutions to McKean's caricature of the nerve equation. I. Existence, Communications on pure and applied mathematics, 41 (1988), pp. 71-103.

[84] - Multiple impulse solutions to McKean's caricature of the nerve equation. II. Stability, Communications on pure and applied mathematics, 41 (1988), pp. 997-1025.

[85] H. R. Wilson, Simplified dynamics of human and mammalian neocortical neurons, Journal of Theoretical Biology, 200 (1999), pp. 375-388.

[86] S. Yi, P. W. Nelson, AND A. G. Ulsoy, Delay differential equations via the Lambert $W$ function and bifurcation analysis: application to machine tool chatter, Mathematical Biosciences and Engineering, 4 (2007), pp. 355-368. 